\title{
Deformation Based Curved Shape Representation
}

\author{
Girum G. Demisse, Djamila Aouada, and Björn Ottersten
}

\begin{abstract}
In this paper, we introduce a deformation based representation space for curved shapes in $\mathbb{R}^{n}$. Given an ordered set of points sampled from a curved shape, the proposed method represents the set as an element of a finite dimensional matrix Lie group. Variation due to scale and location are filtered in a preprocessing stage, while shapes that vary only in rotation are identified by an equivalence relationship. The use of a finite dimensional matrix Lie group leads to a similarity metric with an explicit geodesic solution. Subsequently, we discuss some of the properties of the metric and its relationship with a deformation by least action. Furthermore, invariance to reparametrization or estimation of point correspondence between shapes is formulated as an estimation of sampling function. Thereafter, two possible approaches are presented to solve the point correspondence estimation problem. Finally, we propose an adaptation of k-means clustering for shape analysis in the proposed representation space. Experimental results show that the proposed representation is robust to uninformative cues, e.g. local shape perturbation and displacement. In comparison to state of the art methods, it achieves a high precision on the Swedish and the Flavia leaf datasets and a comparable result on MPEG-7, Kimia99 and Kimia216 datasets.
\end{abstract}

Index Terms-Shape representation, similarity metric, shape matching, deformation.

\section{INTRODUCTION}

$S_{\text {in }}^{\text {Hin }}$ HAPE analysis and modelling finds many applications in computer vision, computational anatomy, and engineering [19], [27], [34], [48]. As a result, different kinds of feature detectors have been designed in an effort to define a robust similarity metric and to train classifiers [7], [60]. However, features are usually finite dimensional and shapes are continuous objects. Hence, in [52] a finite dimensional object is argued to be inadequate to represent an infinite dimensional object, theoretically. Alternatively, shapes can be treated as parametrized functions and a shape space as a Hilbert space, [30], [37], [38], [52], [64]. In general, the parametrization approach leads to a nonlinear and infinite dimensional shape space which, in most cases, is complemented with a metric and treated as a Riemannian manifold. There are several advantages in using the Riemannian framework. The first advantage is the treatment of the shape space as a smooth manifold which is natural considering the nonlinearity of shapes. Secondly, under the Riemannian framework, a smoothly varying metric can be defined on a nonlinear space. Such a metric is essential to describe distance, area, and other geometric notions in a nonlinear space. Lastly, given the Riemannian framework, a nonlinear shape space can be linearised, at least locally, using the notion of geodesic connections. As a result, shape analysis problems can be treated with statistical models. Despite all the described advantages, there are many potential metrics one can define over a nonlinear shape space, leading to different computational and interpenetration challenges. For instance, the $L^{2}$ metric in an infinite dimensional shape space gives a zero distance between different shapes. Con-

- All authors are with the Interdisciplinary Center for Security, Reliability, and Trust of the University of Luxembourg, Luxembourg, L-2721.

E-mail:\{girum.demisse, djamila.aouada, bjorn.ottersten\}@uni.lu

Manuscript received July 19, 2016.

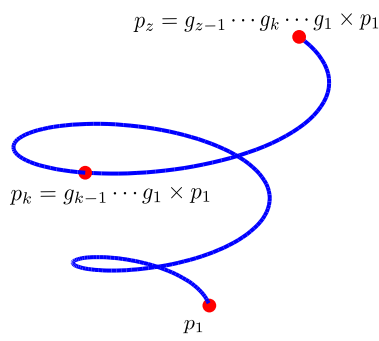

Fig. 1: Illustration of the proposed representation. Given a fixed starting point $p_{1}$, the curve is reconstructed by the successive action of the $\left(g_{1}, \cdots, g_{z-1}\right)$.

sequently, a wide variety of metrics have been proposed in an infinite dimensional shape space [37], [38], [39]. Although some of the proposed metrics have an explicit geodesic equation, some lead to a nontractable geodesic equation [38]. Moreover, almost all of the proposed metrics depend on the differentiability of the considered curved shapes. Thus, self-intersecting shapes or shapes that are not smooth are often excluded. In this paper, we elaborate and extend the deformation based curved shape representation, proposed in [15], that does not impose smoothness constraints while leading to a simple and explicit geodesic equation. ${ }^{1}$ In [15], a new curved shape representation is formulated on the curve's deformation space, which leads to a much simpler metric with an explicit solution to its geodesic equation. The metric has a close relationship with what is sometimes referred to as effort functional [44], [63]. Hence, it measures the cost of optimally deforming one curved shape to another as a distance metric. Moreover,

1. The complete MATLAB code can be found at https://github.com/ DemisseG/Curved_shape_representation 
curves are explicitly represented in the deformation space, which is formulated as a finite dimensional Lie group. Unlike previous deformation based approaches [14], [19], [21], [22], [61], the proposed representation does not refer to a template shape to compute deformations. Instead, the intrinsic characteristics of a deforming curve are represented by finitely many rigid transformation matrices. Intuitively, these transformation matrices capture how a curved shape bends and stretches through space, see Fig. 1. The main advantages of representing curves with the proposed approach are the simplification of the geodesic distance and path between shapes, which are expressed explicitly. Consequently, the optimality and computational cost of the distance computation are completely dependent on the point correspondence estimation method. Furthermore, the group operator of the deformation space can be used to either extract or apply curve deformations, since the curves themselves are represented as elements of the deformation space. Lastly, the induced metric is left invariant and relatively robust to local noise. Nevertheless, there are some drawbacks. The estimation of point correspondence is formulated in terms of computing an optimal sampling function. Hence, the accuracy of the computed geodesic distance is dependent on the sample size (number of points). In general, a large sample size leads to a better performance. This drawback embodies the fundamental assumption of the approach, which is any curved shape can be distinctively described by an arbitrarily large number of discrete points. Computational cost, on the other hand, is a polynomial function of the sample size. The main contributions of this paper are summarized as follows:

1) A relatively simple yet effective representation for curved shapes, described in [15], is introduced. Particular to this representation, curves are treated as elements of the deformation space which is a finite dimensional Lie group. The representation space assumes only continuity and not differentiability. Hence, it is inclusive of curves that are continuous yet not differentiable everywhere.

2) A similarity metric with an explicit geodesic distance that is equivalent to the $L^{2}$-norm is proposed; hence, simplifying distance computation. Furthermore, important practical properties of the metric are discussed and a relationship with a deformation by a least action is established.

3) Given the proposed deformation based representation of curves, two possible approaches for addressing point correspondence estimation are proposed. Characteristics and advantages of one over the other are discussed.

4) The proposed metric is used for learning a fairly simple classifier with k-means clustering.

5) Finally, a comparison of the proposed approach with other shape representation approaches including the square root velocity (SRV) framework [54] is provided on several datasets.

The rest of the paper is organized as follows: in Section 2 an overview of related works is presented followed by a detail description of the proposed shape representation and the metric it induces in Section 3. Properties and interpretations of the metric are presented in Section 4. In
Section 5, two possible approaches for estimating point correspondence between shapes are presented. In Section 6, the metric is used for learning a k-means classifier. Experimental scenarios and results are presented in Section 7. The paper ends with concluding remarks in Section 8.

\section{Related work}

In this section, we present a brief literature review on shape representations and similarity metrics defined on them. For a complete and more extensive survey see [64].

In [28], Kendall defines shape as what remains after removing rotation, translation and uniform scaling. Consequently, almost all shape representations have a long standing goal to be invariant to the mentioned shape preserving transformations. To that end, the pioneering shape representation is a landmark-based shape representation [28]. Landmark-based methods represent a curved shape by a selected set of points. These points are believed to be distinctive of the shape and are called landmarks or features. Subsequently, a curved shape $S$ embedded in $\mathbb{R}^{n}$ and represented by selected $m$ landmarks will be a point in $\mathbb{R}^{m n}$. In such a setting, invariance to translation and scaling is achieved by centring the $m$ vectors to zero mean and scaling $p \in \mathbb{R}^{m n}$ to unit norm [53]. Such a space, according to Kendall, is called pre-shape space. Later on, invariance to rotation is achieved by defining an equivalence relationship between a point $p$ in a pre-shape space and all of its orientations. Thus, the resulting final space, called shape space, is described by modding out the orientations, $\mathbb{R}^{m n} / \mathrm{SO}(\mathrm{n})$. Here, $\mathrm{SO}(\mathrm{n})$ describes a group of $\mathrm{n}$-dimensional rotations. The major drawback of landmark-based approaches is the need for identifying landmarks, which are often selected subjectively. This leads to inconsistent representations, as the same shape can be represented by two completely different sets of landmark points. Nevertheless, in [10], [17], [28], an elegant statistical framework in $\mathbb{R}^{m n} / \mathrm{SO}(\mathrm{n})$ was introduced for landmark based approaches. Alternatively, in [30], [38], [54], [61], curved shapes were represented by a parametrized function leading to an infinite dimensional space. Similar to landmark-based approaches, shape preserving transformations are filtered from the representation space. Furthermore, invariance to reparametrization is achieved by modding out shape preserving diffeomorphisms. In the infinite dimensional setting, a shape space is usually given as $\operatorname{Imm}\left(\mathbb{S}^{1}, \mathbb{R}^{n}\right) / \operatorname{Diff}\left(\mathbb{S}^{1}\right)$, where $\operatorname{Imm}\left(\mathbb{S}^{1}, \mathbb{R}^{n}\right)$ is the space of all parametrized functions immersed in $\mathbb{R}^{n}$ and defined on a 1 -dimensional circle, $\mathbb{S}^{1}$, while $\operatorname{Diff}\left(\mathbb{S}^{1}\right)$ is the group of diffeomorphisms acting on $\mathbb{S}^{1}$. In such a space, the distance between shapes is defined by the length of the geodesic curve connecting them. Consequently, the simplest possible metric is $L^{2}(a, b)=\int\langle a, b\rangle \mathrm{d} s$, where $\langle\cdot, \cdot\rangle$ is a smoothly varying metric tensor, $a$ and $b$ are vector fields tangent to a curve at the shape space and integrated with respect to the arc length. Regardless, the geodesic equation of the $L^{2}$ metric is difficult to solve. Furthermore, a distance function based on the $L^{2}$ metric can, potentially, lead to abnormal results; the distance between two different shapes can be zero [38]. Subsequently, to avoid such abnormalities a first order Sobolev metric was introduced in [37], [38], with numerical solutions. In [54], an isomorphism from 
$\operatorname{Imm}\left(\mathbb{S}^{1}, \mathbb{R}^{n}\right) / \operatorname{Diff}\left(\mathbb{S}^{1}\right)$, with a first order Sobolev metric, to a Hilbert manifold by a mapping function called square root velocity (SRV) was proposed. SRV leads to a geodesic equation with an explicit solution for open curves, while the geodesic distance for closed curves is computed iteratively with a method called path-straightening. In general, in almost all parametrization approaches, shapes are assumed to be $C^{\infty}$ (infinitely differentiable) or at least $C^{2}$, since most metrics in these shape spaces are defined based on differential quantities, e.g., a first order Sobolev metric. Consequently, curves that are self-intersecting and not smooth are excluded. Furthermore, this leads to algorithms that are sensitive to noise, making a pre-smoothing stage a necessity [36].

Alternative to both landmark and parametrization approaches, primarily in the work of Grenander et al. [3], [21], [22], [61], [62], shapes were studied from a different perspective; they were studied based on how they deform from one to another. In [61], a metric defined on a deformation space is shown to induce a distance metric on a shape space, given the deformation space is a group and acts transitively on elements of the shape space. Subsequently, the similarity between two shapes is measured by a deformation that minimizes an objective function called effort functional- the distance between the group's identity and the given deformation, see [61], [63]. Nevertheless, different approaches consider different deformation spaces. In [57], [61], the considered deformation space is a set of $C^{d}$ functions, for some $d \geq 0$. Together with a composition operation, the space of $C^{d}$ functions is shown to be an infinite dimensional Lie group. In this deformation space, elastic shape matching is done by solving a discrete version of the effort functional with dynamic programming. In [22], a general pattern theory that analyses configurations generated by geometric units (lines, points, etc.) based on the deformation from a template configuration was presented. In this case, the considered deformation space is a direct product of matrix Lie groups, see [20] for a brief overview. Recently, in [19], 3D shapes are modelled based on how they deform from a template shape by the action of finite dimensional matrix Lie group. However, in [19], point correspondence is assumed which is not trivial to achieve. In a more similar approach to what is presented here, in [29], the Frenet-Serret frame has been used to represent deformations of a curved shape. However, the Frenet-Serret frame is applicable only to curves with a non-vanishing curvature. Later in [23], a representation where the Frenet-Serret frame is replaced by the action of a general linear group is presented.

In summary, most deformation based approaches follow a paradigm called deformable template, where geometric variations are modelled with respect to a given template shape. In this paper, we present yet another deformation based shape representation without a reference template shape. Consequently, shapes are represented as elements of the deformation space, which in our case is a finite dimensional matrix Lie group. Later in the paper, we will detail similarities between our approach and what was proposed in [61].

\section{Proposed shape Representation}

Let $\overline{\mathcal{S}}$ be a continuous curved shape, and let $\mathcal{S}$ be an approximation of it with $z$ discrete points. For now, we will assume these points to be sampled with uniform arc length interval. In addition to being a set of points, $\mathcal{S}$ carries an order, i.e. $\left(p_{1}, \cdots, p_{z}\right) \neq\left(p_{z}, \cdots, p_{1}\right)$. However, the order and the starting point $p_{1}$ of a shape are selected a priori, for now. Later in the paper, we will discuss the impact of the starting point and the ordering direction; this is similar to what is referred to as reparametrization in the literature [37], [54], [63]. Furthermore, a given curved shape $\mathcal{S}^{1}$ is assumed to be able to deform to another curved shape $\mathcal{S}^{2}$. We denote the space of such shapes by $\mathcal{C}$. However, not all deformations are shape altering. In particular, translation with respect to a fixed frame of reference, uniform scaling and rotation preserve both the shape and the order of points. Consequently, shape preserving deformations cause redundancy in $\mathcal{C}$ and need to be filtered out. Similarly to [28], translation and uniform scaling of a given shape, $\mathcal{S}=\left(p_{1}, \cdots, p_{z}\right)$ where $p_{i} \in \mathbb{R}^{n}$, are filtered out as follows

$$
\mathcal{S}=\left(p_{1}^{*}, \cdots, p_{z}^{*}\right)=\left(\frac{p_{1}-\bar{p}}{h}, \cdots, \frac{p_{z}-\bar{p}}{h}\right),
$$

where $\bar{p}=\frac{1}{z} \sum_{i=1}^{z} p_{i} \in \mathbb{R}^{n}, \quad h=\sqrt{\sum_{i=1}^{z}\left\|p_{i}-\bar{p}\right\|_{2}^{2}} \in \mathbb{R}$, $\|\cdot\|_{2}$ denotes the $L^{2}$-norm. Deformation due to rotation, however, cannot be filtered in a preprocessing stage, see [53] for further details. Consequently, variations due to rotation are identified by an equivalence relationship. Let $R \in \mathrm{SO}(\mathrm{n})$ be an n-dimensional rotation matrix; here, $\mathrm{SO}(\mathrm{n})$ denotes the special orthogonal group. Subsequently, the deformation of a given shape $\mathcal{S}^{j}$ by any $R$ will define an equivalence class $\left[\mathcal{S}^{j}\right]$ in $\mathcal{C}$. Thus, $\left[\mathcal{S}^{j}\right]$ is the set of all shapes that are generated by rotating $\mathcal{S}^{j}$. However, if the correct point correspondence between $\mathcal{S}^{k}$ and $\mathcal{S}^{j}$ is given then $R$ can be computed by optimizing

$$
\underset{R \in \mathrm{SO}(\mathrm{n})}{\arg \min }\left\|R \mathcal{S}^{j}-\mathcal{S}^{k}\right\|_{2}^{2}
$$

Subsequently, we identify a given shape $\mathcal{S} \in \mathcal{C}$ by the following mapping function

$$
f(\mathcal{S})= \begin{cases}\mathcal{G}=\left(g_{1}, \cdots, g_{z}\right) & \text { if } \mathcal{S} \text { is a closed curve, } \\ \mathcal{G}=\left(g_{1}, \cdots, g_{z-1}\right) & \text { if } \mathcal{S} \text { is an open curve }\end{cases}
$$

such that

$$
g_{i} \times p_{i}=p_{i+1},
$$

where $g_{i=1, \cdots, z}$ are transformation matrices that map one point to another with respect to a fixed coordinate frame. In this work, we will only consider Euclidean transformations without reflection and thus $\mathcal{G}$ is an element of $\mathrm{SE}(\mathrm{n})^{z}$, which is a direct product of a Special Euclidean group. Although (4) is underdetermined, in this work, the $g_{i=1, \cdots, z}$ refer to optimal transformations that preserve the fixed global frame, see Appendix on computational details. Intuitively, the representation $f(\cdot)$ attempts to encode every curve $\mathcal{S} \in \mathcal{C}$ as the path of a travelling particle from $a$ 
fixed starting point, see Fig. 1. The inverse of the mapping function, for closed curves, is defined as

$$
f^{-1}(\mathcal{G})=\left(p_{1}, g_{1} p_{1}, g_{2} g_{1} p_{1}, \cdots,\left(\prod_{i=1}^{z} g_{i}\right) p_{1}\right) .
$$

The inverse for open curves can be defined similarly. Moreover, the representation $f(\cdot)$ also preserves the equivalence relationship defined in $\mathcal{C}$.

Proposition 1. If $\mathcal{G}^{a}$ and $\mathcal{G}^{b}$ are the representations of $\mathcal{S}^{a}, \mathcal{S}^{b} \in$ $\left[\mathcal{S}^{j}\right]$ then $\mathcal{G}^{a}$ is equivalent to $\mathcal{G}^{b}$ by conjugacy, $\mathcal{G}^{a} \sim \mathcal{G}^{b}$.

Proof. Let $\mathcal{S}^{a}=\left(p_{1}^{a}, \cdots, p_{z}^{a}\right)$ and $\mathcal{S}^{b}=\left(p_{1}^{b}, \cdots, p_{z}^{b}\right)$. Since, $\mathcal{S}^{a}, \mathcal{S}^{b} \in\left[\mathcal{S}^{j}\right]$ we can write $\mathcal{S}^{b}=R \mathcal{S}^{a}$, where $R \in \mathrm{SO}(\mathrm{n})$. Rotation is an isometry, thus $R \mathcal{S}^{a}=\left(R p_{1}^{a}, \cdots, R p_{z}^{a}\right)$. The representation $f\left(\mathcal{S}^{b}\right)=\left(g_{1}^{b}, \cdots, g_{z}^{b}\right)$, such that

$$
\begin{aligned}
g_{i}^{b} \times p_{i}^{b} & =p_{i+1}^{b} \\
& =R \times p_{i+1}^{a},
\end{aligned}
$$

can then be expressed in terms of $f\left(\mathcal{S}^{a}\right)$ as follows

$$
\begin{aligned}
g_{i}^{b} \times R \times p_{i}^{a} & =R \times g_{i}^{a} \times p_{i}^{a} \\
g_{i}^{b} & =R \times g_{i}^{a} \times R^{-1} .
\end{aligned}
$$

Thus, $f\left(\mathcal{S}^{a}\right)$ and $f\left(\mathcal{S}^{b}\right)$ are equivalent by conjugacy, i.e., $f\left(\mathcal{S}^{b}\right)=R f\left(\mathcal{S}^{a}\right) R^{-1}$.

As a result, the equivalence relationship defined in $\mathcal{C}$ carries over to the representation space $\mathrm{SE}(\mathrm{n})^{z}$. Computationally, if two given shapes belong to the same shape class then the corresponding eigenvalues of the transformation matrices in $f\left(\mathcal{S}^{b}\right)$ and $f\left(\mathcal{S}^{a}\right)$ are similar. Finally, the representation space of equivalent classes is described by modding out the rotations as $\mathrm{SE}(\mathrm{n})^{z} / \mathrm{SO}(\mathrm{n})$, for closed curves, and $\mathrm{SE}(\mathrm{n})^{z-1} / \mathrm{SO}(\mathrm{n})$, for open curves. Note that the representation space still assumes point correspondence or parametrization. Thus, neither $\mathrm{SE}(\mathrm{n})^{z} / \mathrm{SO}(\mathrm{n})$ nor $\mathrm{SE}(\mathrm{n})^{z-1} / \mathrm{SO}(\mathrm{n})$ are invariant to reparametrization; we will address this issue in Section 5. Moreover, any given shape that is represented in $\mathrm{SE}(\mathrm{n})^{z} / \mathrm{SO}(\mathrm{n})$ is deformable to any other representation in $\mathrm{SE}(\mathrm{n})^{z} / \mathrm{SO}(\mathrm{n})$.

Let $f\left(\mathcal{S}^{1}\right)$ be the representation of $\mathcal{S}^{1}$ that we want to deform to $\mathcal{S}^{2}$ represented by $f\left(\mathcal{S}^{2}\right)$. Given the point correspondence between $\mathcal{S}^{1}$ and $\mathcal{S}^{2}$, we can extract the deformation of $f\left(\mathcal{S}^{1}\right)$ to $f\left(\mathcal{S}^{2}\right)$ as

$$
\mathcal{G}^{L}=f\left(\mathcal{S}^{2}\right) f\left(\mathcal{S}^{1}\right)^{-1},
$$

so that $\mathcal{G}^{L} f\left(\mathcal{S}^{1}\right)=f\left(\mathcal{S}^{2}\right)$. Note that $f\left(\mathcal{S}^{1}\right)^{-1}$ is element wise inversion of the representation, unlike $f^{-1}\left(\mathcal{S}^{1}\right)$ which is a map from the representation space to $\mathcal{C}$. Since $\mathrm{SE}(\mathrm{n})^{z} / \mathrm{SO}(\mathrm{n})$ is a group, the deformation $\mathcal{G}^{L}$ is an element of $\mathrm{SE}(\mathrm{n})^{z} / \mathrm{SO}(\mathrm{n})$ acting from the left. Alternatively, a deformation that acts from the right is defined as

$$
\mathcal{G}^{R}=f\left(\mathcal{S}^{1}\right)^{-1} f\left(\mathcal{S}^{2}\right) .
$$

Henceforth, we will use $\mathcal{G}^{L}$ and $\mathcal{G}^{R}$ to describe deformations that act from the left and deformations that act from the right, respectively. Consequently, the deformation space under consideration is the same as the proposed shape representation space.

\subsection{Distance in $\mathrm{SE}(\mathrm{n})^{z}$}

The proposed representation space $\mathrm{SE}(\mathrm{n})^{z}$ is a matrix Lie group, which is not a Euclidean space. As a result, the usual definition of distance as a straight line does not generalize to $\mathrm{SE}(\mathrm{n})^{z}$. Alternatively, techniques from differential geometry can be used to define distance in a curved space. In this subsection, we will overview concepts from Lie theory and differential geometry to later develop a distance function in $\mathrm{SE}(\mathrm{n})$, and its direct product group $\mathrm{SE}(\mathrm{n})^{z}$.

A Lie group is a smooth manifold with smooth group operations; that is, the group's binary operator $(x, y) \mapsto x y^{-1}$ is $C^{\infty}$. The tangent space at the identity of the group $e$ is an algebra called Lie algebra, which we will denote by $\mathfrak{g}$. The smooth and invertible binary operator of a Lie group enables one to define a diffeomorphism onto itself. For instance, consider a left translation of a Lie group defined as $L_{a}: G \rightarrow a G, a \in G$, where $G$ denotes the Lie group. Meanwhile, the differential structure, due to Lie groups being smooth manifolds, enables one to do calculus on a Lie group. To compute distance, volume and other geometric notions, however, an additional structure called metric is needed. Consequently, a Lie group $G$ can be complemented with a smoothly varying metric tensor $\langle\cdot, \cdot\rangle$, making it a Riemannian manifold. The metric tensor $\langle\cdot, \cdot\rangle$ is defined at the tangent space $T_{g} G$ as $\langle\cdot, \cdot\rangle_{g}: T_{g} G \times T_{g} G \rightarrow \mathbb{R}$ for every $g \in G$, see [16], [42] for a detailed discussion. Moreover, a metric tensor on a Lie group is said to be left invariant if the left translation diffeomorphism is an isometery, i.e., if the following is true

$$
\langle x, y\rangle_{e}=\left\langle d L_{a} x, d L_{a} y\right\rangle_{a}, \quad \forall x, y \in \mathfrak{g}, \forall a \in G,
$$

where $d L_{a}$ is the derivative of the left translation $L_{a}$. As a result, a left invariant Riemannian metric is identified with $\langle\cdot, \cdot\rangle_{e}$ through the pullback map, $d L_{a}^{-1}$. Subsequently, the length of a given curve $\gamma$ defined on a subset of $\mathbb{R}$, $\gamma:\left[t_{0}, t_{1}\right] \rightarrow G$, is given as

$$
\ell(\gamma)=\int_{t_{0}}^{t_{1}} \sqrt{\langle\dot{\gamma}(t), \dot{\gamma}(t)\rangle_{\gamma(t)}} \mathrm{d} t
$$

where $\dot{\gamma}(\cdot) \in T_{\gamma(t)} G$ is the derivative of the curve. There are several possible curves that start $\gamma\left(t_{0}\right) \in M$ and end at $\gamma\left(t_{1}\right) \in M$; the shortest curve among them is called geodesic curve. The distance between $\gamma\left(t_{0}\right)$ and $\gamma\left(t_{1}\right)$ is defined as the length of the geodesic curve

$$
d\left(\gamma\left(t_{0}\right), \gamma\left(t_{1}\right)\right)=\operatorname{Inf}\{\ell(\gamma)\} .
$$

The derivatives of a given curve $\gamma$ define a vector field on the manifold $\dot{\gamma}$. More interestingly, if a vector field $\dot{\gamma}(h)$ defined on a Lie group is left invariant, i.e., if the following is true for $h \in G$

$$
d L_{a} \dot{\gamma}(h)=\dot{\gamma}(a h) \in T_{a h} G,
$$

then it is parallel along the curve, and its integral curve $\gamma(t)=\exp (t \dot{\gamma})$ is geodesic. If any two points on a manifold can be connected by a geodesic curve then the manifold is called geodesically complete or compact.

Similarly, geodesic curves and the geodesic distance can be defined in $\mathrm{SE}(\mathrm{n})$. However, $\mathrm{SE}(\mathrm{n})$ is not a compact group but a semidirect product of a compact group, $\mathrm{SO}(\mathrm{n})$, and 
$\mathbb{R}^{n}$, which can be described in a homogeneous coordinates as follows

$$
g_{i}=\left(\begin{array}{cc}
R_{i} & v_{i} \\
0 & 1
\end{array}\right), \text { s.t., } R_{i} \in \mathrm{SO}(\mathrm{n}), v_{i} \in \mathbb{R}^{n} .
$$

Subsequently, geodesic curves that connect two points in $\mathrm{SO}(\mathrm{n})$ and $\mathbb{R}^{n}$, respectively, are defined as

$$
\begin{aligned}
\varphi_{R}(t) & =R_{1}\left(R_{1}^{-1} R_{2}\right)^{t} \\
\varphi_{v}(t) & =v_{1}+\left(v_{2}-v_{1}\right) t,
\end{aligned}
$$

where $t \in[0,1]$. It can easily be checked that $\varphi_{R}(t)$ is geodesic in $\mathrm{SO}(\mathrm{n})$, though, not necessarily unique [8], [41], whereas $\varphi_{v}(t)$ is clearly geodesic since $\mathbb{R}^{n}$ is a vector space. Consequently, in [65] the following curve in $\mathrm{SE}(\mathrm{n})$ is proven to be geodesic.

$$
\varphi(t)=\left(\begin{array}{cc}
\varphi_{R}(t) & \varphi_{v}(t) \\
0 & 1
\end{array}\right)
$$

where $t \in[0,1]$. Moreover, we can define a Riemannian metric on the Lie algebra of SE(n) as

$$
\left\langle\left(\mathfrak{R}_{1}, v_{1}\right),\left(\mathfrak{R}_{2}, v_{2}\right)\right\rangle_{e}=\left\langle\mathfrak{R}_{1}, \mathfrak{R}_{2}\right\rangle+\left\langle v_{1}, v_{2}\right\rangle,
$$

where $\mathfrak{R} \in \mathfrak{s o}(n)$, the Lie algebra of $\mathrm{SO}(\mathrm{n})$. Subsequently, using (15) and (16), the length of a geodesic curve connecting $g_{1}, g_{2} \in \mathrm{SE}(\mathrm{n})$ can be computed by transporting the tangent vectors with the pullback to the Lie algebra. The geodesic distance, in this case, reads as

$$
d\left(g_{1}, g_{2}\right)=\int_{0}^{1} \sqrt{\left\langle d L_{\varphi(t)}^{-1}(\dot{\varphi}(t)), d L_{\varphi(t)}^{-1}(\dot{\varphi}(t))\right\rangle_{e}} \mathrm{~d} t .
$$

Since $\varphi(t)$ is a geodesic curve, the tangent vectors $\dot{\varphi}(t)$ are parallel along $\varphi(t)$. Hence, the geodesic distance given in (17) is simplified as

$$
\begin{aligned}
d\left(g_{1}, g_{2}\right) & =(1-0) \times\langle\dot{\varphi}(t), \dot{\varphi}(t)\rangle_{e} \\
& =\left(\left\|\log \left(R_{1}^{T} R_{2}\right)\right\|_{F}^{2}+\left\|v_{2}-v_{1}\right\|_{2}^{2}\right)^{1 / 2},
\end{aligned}
$$

where $\|\cdot\|_{F}$ denotes the Frobenius norm. Subsequently, we extend the geodesic curve $\varphi(t)$ given in (15) to the direct product space $\mathrm{SE}(\mathrm{n})^{z}=\mathrm{SE}(\mathrm{n})_{1} \times \cdots \times \mathrm{SE}(\mathrm{n})_{z}$ as follows

$$
\zeta\left(\mathcal{G}_{1}, \mathcal{G}_{2}\right)=\left(\varphi(t)_{1}, \cdots, \varphi(t)_{z}\right),
$$

such that $\varphi(t)_{i}$ is the geodesic curve between $g_{i}^{1} \in \mathcal{G}_{1}$ and $g_{i}^{2} \in \mathcal{G}_{2}$. It can be shown that (19) is a geodesic curve in the product group, see [16]. A geodesic distance in $\mathrm{SE}(\mathrm{n})^{z}$ follows directly from (19) and is given as

$$
\mathfrak{d}\left(\mathcal{G}_{1}, \mathcal{G}_{2}\right)=\left(d\left(g_{1}^{1}, g_{1}^{2}\right)^{2}+\cdots+d\left(g_{z}^{1}, g_{z}^{2}\right)^{2}\right)^{1 / 2} .
$$

In summary, the geodesic path and distance between the representations of two shapes $f\left(\mathcal{S}^{1}\right)$ and $f\left(S^{2}\right)$ can be computed using (19) and (20), respectively. Nevertheless, the defined geodesic distance (20) is not invariant to parametrization.

\section{Properties and interpretation}

In this section, we present some of the important properties of the proposed representation and its metric followed by a discussion on its interpretation and relationship with earlier works.
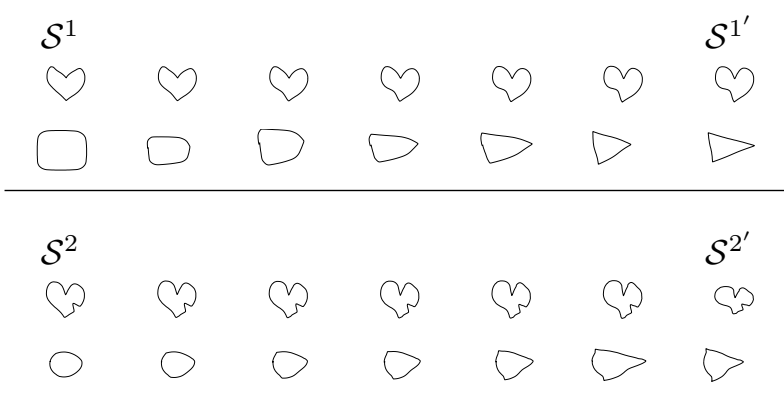

Fig. 3: The first set of shapes shows two examples where $\mathcal{S}^{1}$ deforms to $\mathcal{S}^{1^{\prime}}$. The second set shows the transported deformation to their similar objects $\mathcal{S}^{2}$ to give $\mathcal{S}^{2^{\prime}}$, respectively.

\subsection{Properties}

1) The proposed distance metric is left invariant. In general, a metric $\rho$ that is defined on a group $G$ is said to be left invariant if $\rho(a, b)=\rho(q a, q b), \forall a, b, q \in G$, and the defined metric (20) is left invariant see [40] for further details. The implication of this is that the distance between two shapes is invariant to a deformation acting on both shapes from the left. More concretely, let $\mathcal{G}^{\prime} \in \mathrm{SE}(\mathrm{n})^{z}$ be a deformation acting on the representations of two distinct shapes, $f\left(\mathcal{S}^{1}\right)$ and $f\left(\mathcal{S}^{2}\right)$, then $\mathfrak{d}\left(f\left(\mathcal{S}^{1}\right), f\left(\mathcal{S}^{2}\right)\right)=\mathfrak{d}\left(\mathcal{G}^{\prime} f\left(\mathcal{S}^{1}\right), \mathcal{G}^{\prime} f\left(\mathcal{S}^{2}\right)\right)$. This fact can be observed by plugging the action of $\mathcal{G}^{\prime}$ into (20), in which case it will cancel itself out. As discussed in [15], this property is particularly important in transporting deformation between two similar shapes. To clarify further, we consider below a deformation transportation problem discussed in [54]. Let $\mathcal{S}^{1}$ and $\mathcal{S}^{1^{\prime}}$ be shape contours representing exactly the same real world object denoted by $O^{1}$, except that $\mathcal{S}^{1^{\prime}}$ is deformed under some unknown external factor. For instance, $\mathcal{S}^{1}$ and $\mathcal{S}^{1^{\prime}}$ can be contours of $O^{1}$ from a different viewing angle. Furthermore, let $O^{2}$ denote a similar, but not identical, object to $O^{1}$ with $\mathcal{S}^{2}$ as its shape contour. Subsequently, given $\mathcal{S}^{1}, \mathcal{S}^{1^{\prime}}$, and $\mathcal{S}^{2}$ the problem of estimating $\mathcal{S}^{2^{\prime}}$, a deformed $\mathcal{S}^{2}$ under the same external factor, can be framed as a deformation transportation problem. In [54], the deformation is estimated by transporting the vector field along the geodesic curve connecting $\mathcal{S}^{1}$ and $\mathcal{S}^{1}$. Alternatively in our framework, the deformation due to an external factor can be factored out as $\mathcal{G}^{L}=f\left(\mathcal{S}^{1^{\prime}}\right) f\left(\mathcal{S}^{1}\right)^{-1}$. Consequently, since our metric is left invariant, $\mathfrak{d}\left(f\left(\mathcal{S}^{1}\right), f\left(\mathcal{S}^{2}\right)\right)=\mathfrak{d}\left(\mathcal{G}^{L} f\left(\mathcal{S}^{1}\right), \mathcal{G}^{L} f\left(\mathcal{S}^{2}\right)\right)$. Thus, $f\left(\mathcal{S}^{2^{\prime}}\right)=\mathcal{G}^{L} f\left(\mathcal{S}^{2}\right)$, see Fig. 3 .

2) The proposed metric does not compute differential quantities of curved shapes. Most infinite dimensional representations define a similarity metric based on differential quantities [37], [54]. However, differential quantities are highly sensitive to noise and local perturbations. Consequently, a pre-smoothing stage is necessary before analysing a shape. On the other hand, pre-smoothing a shape can potentially filter legitimate features, see Fig. 2. Although the proposed representation is based on the relative transformation matrices between neighbouring points, it is not as severely sensitive as curvature is, for example, to local perturbations [36]. Hence, needs no pre-smoothing.

3) The proposed representation encodes a given curve by the relative deformation of points as opposed to absolute deforma- 


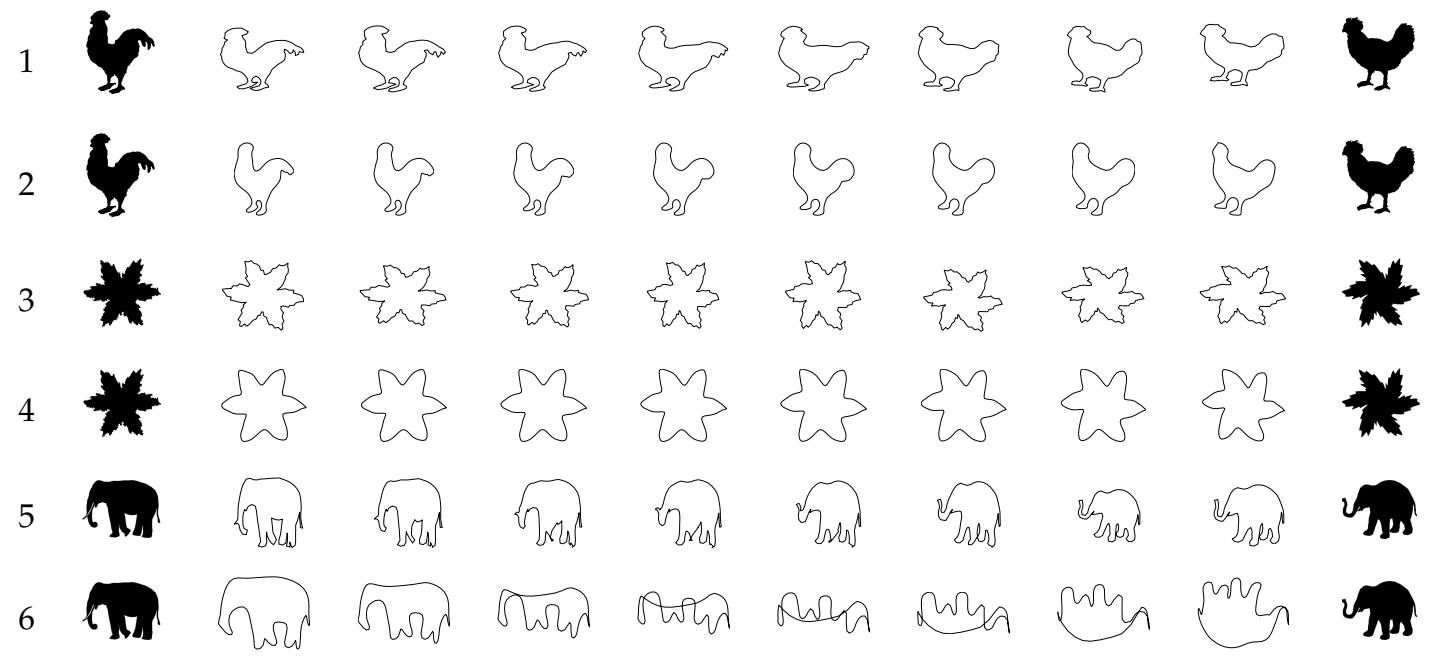

Fig. 2: Shapes along the geodesic path between the initial shape (first column) and target shape (last column). The odd rows show results from our approach while the even rows are results from [54]. All shapes are represented by 100 uniformly sampled and normalized points. We note that results from [54] are smoothed and loses local features of the shapes.

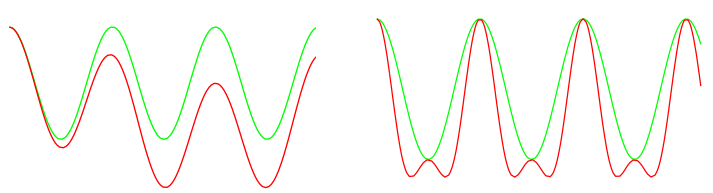

Fig. 4: Two pairs of curves. Under AD, the second pair of curves are more similar than the first, while under RD the first pair are more similar than the second.

tion of points. To further elaborate, let $\mathcal{S}^{1}=\left(p_{1}^{1}, \cdots, p_{z}^{1}\right)$ and $\mathcal{S}^{2}=\left(p_{1}^{2}, \cdots, p_{z}^{2}\right)$ be two shapes that are scaled and aligned, see Fig. 4. Subsequently, we define absolute deformation of points (AD) as the transformation of every $p_{i}^{1} \in \mathcal{S}^{1}$ to match its corresponding point $p_{i}^{2} \in \mathcal{S}^{2}$. In earlier works, such deformations are modelled by displacement vector fields [2] and in some cases by the action of a matrix Lie group [19]. If we formulate the matching of each point, from $\mathcal{S}^{1}$ to $\mathcal{S}^{2}$, by the action of rigid transformation matrices then the $\mathrm{AD}$ is given by $\mathcal{G}=\left(T_{1}, \cdots, T_{z}\right) \in \mathrm{SE}(\mathrm{n})^{z}$ such that

$$
\mathcal{S}^{2}=\mathcal{G S}^{1}=\left(T_{1} p_{1}, \cdots, T_{z} p_{z}\right)
$$

Alternatively, rather than aligning every matching point, as is done with $\mathrm{AD}$, one can align the intrinsic properties of the curves- these are properties of a curve that are mainly invariant to displacement. In this paper, intrinsic properties of a given curve are approximated by the relative deformation of the curve's points, as defined in (3) by $f(\cdot)$. Hence, alignment of $\mathcal{S}^{1}$ and $\mathcal{S}^{2}$ under relative deformation of points $(\mathrm{RD})$ is given as $f\left(\mathcal{S}^{2}\right)=f\left(\mathcal{S}^{1}\right) \mathcal{G}^{R}$, where $\mathcal{G}^{R} \in \mathrm{SE}(\mathrm{n})^{z}$, see Section 3. Subsequently, using (18) the cost of deformation under $\mathrm{RD}$ is given by

$$
\begin{aligned}
d_{r}\left(\mathcal{S}^{1}, \mathcal{S}^{2}\right) & =\left(\sum_{i=1}^{z} d\left(e, g_{i}^{R}\right)\right)^{1 / 2} \\
& =\left(\sum_{i=1}^{z}\left\|\log \left(\left(R_{i}^{1}\right)^{T} R_{i}^{2}\right)\right\|_{F}^{2}+\left\|v_{i}^{2}-v_{i}^{1}\right\|_{2}^{2}\right)^{1 / 2} .
\end{aligned}
$$

\subsection{Interpretation}

The proposed distance function, as derived in (20), has a direct relationship to what is called effort functional [57], [61], [63]. In general, distance between shapes based on effort functional is written as

$$
d_{s}\left(\overline{\mathcal{S}}^{1}, \overline{\mathcal{S}}^{2}\right)=\min _{\psi \in \Psi}\left\{d_{\Psi}(e, \psi): \overline{\mathcal{S}}^{1}=\psi\left(\overline{\mathcal{S}}^{2}\right)\right\}
$$

where $\Psi$ is the space of allowed deformations for matching $\overline{\mathcal{S}}^{2}$ to $\overline{\mathcal{S}}^{1}, d_{s}$ and $d_{\Psi}$ are distance metrics in the shape and deformation spaces, respectively. Such formulation leads to a natural interpretation of distance as a measure of deformation by least action, hence the term effort functional. For further mathematical details see [45], [57]. Similarly, a version of (24) under the proposed representation can be formulated by explicitly defining the space of deformations $\Psi$ for matching $\overline{\mathcal{S}}^{1}$ and $\overline{\mathcal{S}}^{2}$. To define $\Psi$ more precisely, we introduce monotonic and injective maps from a discrete parameter space to the arc length parametrization as $\xi_{i}:[1, z] \rightarrow[0, \ell]$. Here, $\ell$ denotes the length of a curve, $z$ and $i$ are variables for any positive integer, where the latter is used as an identifier for different mappings. Given a fixed $z$, the mappings have a similar effect as to what are referred as shape and orientation preserving diffeomorphisms [63], although in this case there is no guarantee for a mapping to 

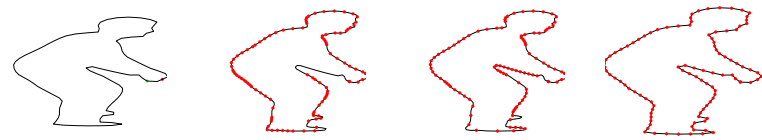

$\overline{\mathcal{S}}$

$\overline{\mathcal{S}} \circ \xi_{2}$

$\overline{\mathcal{S}} \circ \xi_{*}$
Fig. 5: Different samplings of a given shape $\overline{\mathcal{S}}$. The red dots denote sampled points. The last sampling $\xi_{*}$ is a uniform sampling. Note that $\xi_{1}$ and $\xi_{2}$ do not preserve shape.

preserve shape, see Fig. 5. In other words, the mappings are sampling functions that select $z$ ordered points from a continuous curve. Subsequently, $\mathcal{S}_{i}^{j}=\overline{\mathcal{S}}^{j} \circ \xi_{i}$ denotes ordered sample points from $\overline{\mathcal{S}}^{j}$ obtained by using $\xi_{i}$. Finally, we define the set of deformations between $\overline{\mathcal{S}}^{1}$ and $\overline{\mathcal{S}}^{2}$, for a given $z$, as $\Psi=\left\{\mathcal{G}^{R} \mid \exists \xi_{i}, \xi_{j}: f\left(\overline{\mathcal{S}}^{1} \circ \xi_{i}\right) \mathcal{G}^{R}=f\left(\overline{\mathcal{S}}^{2} \circ \xi_{j}\right)\right\}$. Consequently, under the framework of the proposed representation, distance between two given shapes is given as

$$
d_{s}\left(\overline{\mathcal{S}}^{1}, \overline{\mathcal{S}}^{2}\right)=\min _{\mathcal{G}^{R} \in \Psi} \mathfrak{d}\left(f\left(\mathcal{S}_{i}^{1}\right), f\left(\mathcal{S}_{j}^{2}\right)\right) .
$$

Moreover, using the left invariance property of the metric and the definition of $\Psi$, (25) can be simplified as follows

$$
d_{s}\left(\overline{\mathcal{S}}^{1}, \overline{\mathcal{S}}^{2}\right)=\min _{\mathcal{G}^{R} \in \Psi} \mathfrak{d}\left(f\left(\mathcal{S}_{i}^{1}\right), f\left(\mathcal{S}_{i}^{1}\right) \mathcal{G}^{R}\right)=\mathfrak{d}\left(e, \mathcal{G}^{R}\right) .
$$

Hence, a version of the effort functional (24) is described as (26) in the proposed representation space. However, if $\xi_{i}$ and $\xi_{j}$ are given a priori, $\Psi$ will be a singletonsince $\mathcal{G}^{R}$ can be computed directly, as described in (7). For instance, we have so far assumed a perfect point-topoint correspondence between shapes. Thus, $\xi_{i}$ and $\xi_{j}$ are assumed to be known. Consequently, the proposed metric can be viewed as measuring the optimal deformation between two shapes given optimal point correspondence. In this context, optimal deformation is to be interpreted as the deformation with the least action. While, optimal point correspondence stands for the estimation of $\xi_{i}$ and $\xi_{j}$ such that (25) is minimized.

\section{Point CorRespondence estimation}

In general, point correspondence estimation is given by the solution of the following

$$
\underset{\psi \in \Psi}{\arg \min }\left\{d_{s}\left(\psi\left(\overline{\mathcal{S}}^{1}\right), \overline{\mathcal{S}}^{2}\right)+E(\psi)\right\},
$$

where $\Psi$ denotes a deformation space, $d_{s}$ is a dissimilarity metric, and $E(\cdot)$ is an energy term that measures the cost of the deformation. The objective of (27) is to solve not only for optimal point matching but for smooth deformations as well. There are several works in the formulation and optimization of both $d_{s}$ and $E(\cdot)$. In the deformation based approaches, the energy term is usually described in terms of the effort functional given in (24) [61]. However, there are several elaborate formulations of the energy term based on elasticity theory [45], [63]. Similarly, extensive work has been done in formulating a dissimilarity function. Particular to shape matching, in [12] a dissimilarity function that is based on curvature is formulated. Later, in [55] and [50], a symmetric version of what was proposed in [12] is presented and solved with dynamic programming. Regardless of the dissimilarity function, in [49] it is shown that restricting the matching solutions to those that preserve order of points leads to a more accurate result than the unrestricted case. In this section, we formulate point correspondence estimation problem as an estimation of sampling function and propose two possible approaches to solve it. The general form of shape matching cost (27) is given as a measure of shape similarity and deformation cost. However, as shown in (26), the proposed similarity metric is equivalent to effort functional. Hence, it measures the cost of deformation as a dissimilarity between shapes. Subsequently, since $\mathcal{G}^{R}=f\left(\mathcal{S}_{i}^{1}\right)^{-1} f\left(\mathcal{S}_{j}^{2}\right)$ we describe (26) in terms of the sampling functions as

$$
\min _{\xi_{i}, \xi_{j}} \mathfrak{d}\left(e, f\left(\mathcal{S}_{i}^{1}\right)^{-1} f\left(\mathcal{S}_{j}^{2}\right)\right) .
$$

However, the cost functional given in (28) assumes the sampling functions preserve shape. On the contrary, if the sampling function does not preserve shape then (28) will deviate from the target shape, see Fig. 5. In this work, instead of insisting on sampling functions that preserve shape we add a penalty term on (28) so that we get a constrained objective functional that attempts to balance optimal point matching and shape preservation. To that end, let $\xi_{*}$ denote a uniform sampler and assume that it preserves shape. Subsequently, the alteration of a given shape $\overline{\mathcal{S}}^{1}$, when sampled by $\xi_{i}$, from a uniformly sampled one is quantified as

$$
\rho_{\overline{\mathcal{S}}^{1}}\left(\xi_{*}, \xi_{i}\right)=\sum_{j=2}^{z} A_{\overline{\mathcal{S}}^{1}}\left(p_{j-1}^{*}, p_{j}^{*}\right)-\sum_{j=2}^{z} A_{\overline{\mathcal{S}}^{1}}\left(p_{j-1}^{i}, p_{j}^{i}\right) .
$$

Each term of (29) computes the enclosed area of $\overline{\mathcal{S}}^{1}$ when sampled by $\xi_{*}$ and $\xi_{i}$ using Green's theorem. Note that the curves are oriented. Consequently, $\rho_{\overline{\mathcal{S}}^{1}}(\cdot, \cdot)$ is used to penalize a sampler that does not preserve area- in case of non-planar curves one can replace the area preservation requirement with length preservation requirement which is less restrictive. Subsequently, the constrained point correspondence cost functional is given as

$$
\min _{\xi_{i}, \xi_{j}}\left\{\alpha \times \mathfrak{d}\left(e, f\left(\mathcal{S}_{i}^{1}\right)^{-1} f\left(\mathcal{S}_{j}^{2}\right)\right)+\beta \times\left(\rho_{\overline{\mathcal{S}}^{1}}\left(\xi_{*}, \xi_{i}\right)+\rho_{\overline{\mathcal{S}}^{2}}\left(\xi_{*}, \xi_{j}\right)\right)\right\} .
$$

We have added weighting factors $\alpha$ and $\beta$ to emphasize the effect of one over the other- assigning a large value for $\beta$ leads to an objective functional that prefers samplers that preserve area even with a high deformation cost and vice versa for a small $\beta$ value, see Fig. 7. The objective functional given in (30) is symmetric. Moreover, its solution can be estimated via dynamic programming, since it can be decomposed into linearly sequential sub-problems [6]. Subsequently, we propose two possible methods for estimating point correspondence between shapes based on (30). In the next subsections, we will consider the optimization of (30) with dynamic programming, and later we will consider its optimization constrained to uniform sampling.

\subsection{Optimal sampling}

In this subsection, we will describe a dynamic programming based solution for optimal point correspondence estimation 


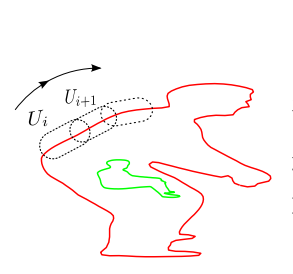

(a)

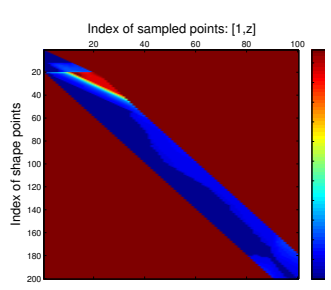

(b)

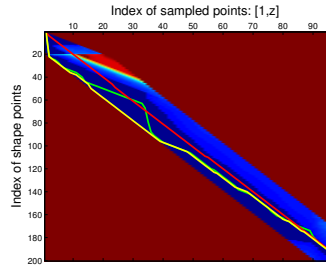

(c) $\alpha=1, \beta=10$

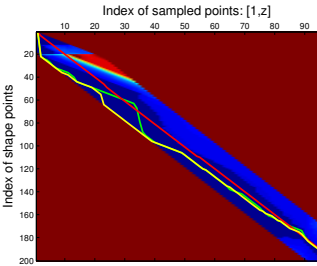

(d) $\alpha=1, \beta=1$

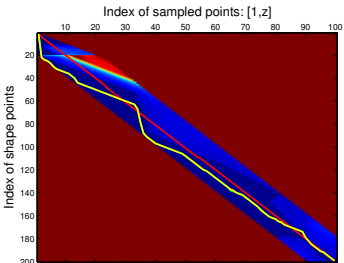

(e) $\alpha=10, \beta=1$

Fig. 6: Intermediate values of dynamic programming. (a) shows two input shapes. $\mathcal{S}_{*}^{1}$, coloured in green, is uniformly sampled and $\overline{\mathcal{S}}^{2}$, coloured in red, is to be sampled optimally. (b) shows the search space, defined by the charts $U_{i}$, and the cost of selecting a point from $\overline{\mathcal{S}}^{2}$ for the $i^{\text {th }}$ position with the color coding. (c-e) shows three optimal sampling paths based on (32) for different values of $\alpha$ and $\beta$. The green path is an optimal for $\alpha=1$ and $\beta=0$, the red path is an optimal for $\alpha=0$ and $\beta=1$, and finally the yellow path is an optimal sampling for $\alpha$ and $\beta$ values shown below the figures.

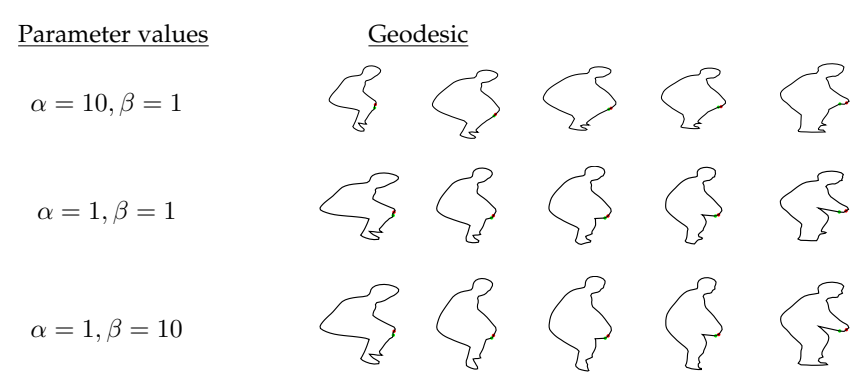

Fig. 7: Geodesic paths for different values of $\alpha$ and $\beta$. Note the impact of a large $\beta$ value on the deformation (geodesic) and the final result.

between open curves. Later, we will address the case of closed curves. As indicated earlier, the objective functional given in (30) can be solved with dynamic programming in a polynomial time. To show this fact, we will first describe $z$ sample points of a shape in a more general form as

$$
\overline{\mathcal{S}}^{k} \circ \xi_{j}=\left(x_{1}, x_{2}, \cdots, x_{z}\right): \quad x_{i} \in U_{i},
$$

where $x_{i=1, \cdots, z}$ are variables that can take points as a value from their respective domain $U_{i=1, \cdots, z}$, while the $U_{i} \subset \overline{\mathcal{S}}^{k}$ are charts of the curve $\overline{\mathcal{S}}^{k}$. Hence, the size of $U_{i}$ introduces a restriction on the search space of the corresponding $x_{i}$. In this work, we define the charts $U_{i=1, \cdots, z}$ by sliding a pre-defined window size to cover the whole curve, see Fig. 6a. The ratio of the window sizes, defined on two given shapes, determines the constraint on the elasticity of a deformation from one to another, we denote this ratio by $\eta$. For instance, If $w_{1}$ and $w_{2}$ are the window sizes defined for $\overline{\mathcal{S}}^{1}$ and $\overline{\mathcal{S}}^{2}$, respectively, then we approximate the elasticity constraint by $\eta=w_{2} / w_{1}$, see Fig. 8. Moreover, we fix the sampling of one of the argument shapes to uniform sampling. Fixing one of the samplings converts what was a symmetric objective functional (30) to asymmetric one. This is computationally efficient, when aligning a set of shapes, e.g., with a fixed reference shape $n$-shapes requires $n-1$ alignment operations, whereas a direct one-to-one alignment requires $(n-1)$ ! alignment operations. Moreover, we have not seen a significant variation in performance due to the choice of a particular curve as a reference, see results in Subsection 7.1 where each shape is used as a reference.

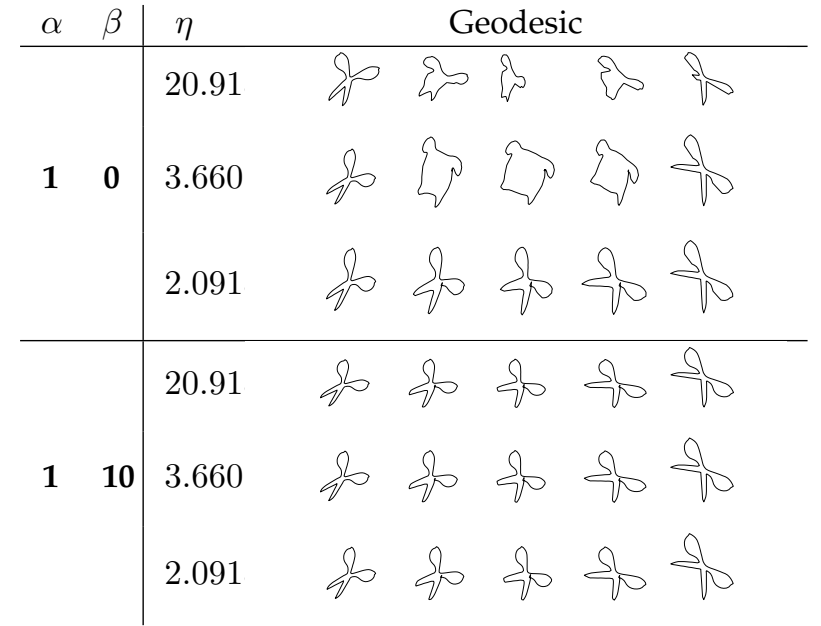

Fig. 8: Geodesic paths between two shapes under different elasticity constraint $\eta$. Note that an objective functional with an appropriate $\beta$ value gives a consistent result regardless of the elasticity constraint.

Subsequently, given a uniformly sampled reference curve $\mathcal{S}_{*}^{1}$, let its representation be $f\left(\mathcal{S}_{*}^{1}\right)=\left(g_{1}^{*}, \cdots, g_{z}^{*}\right)$. Subsequently, we solve for optimal sampler of a given curve $\overline{\mathcal{S}}^{2}$, with respect to $\mathcal{S}_{*}^{1}$, by optimizing (30), which is rewritten as

$$
\underset{\xi_{j}}{\arg \min } \sum_{i=2}^{z} \phi_{i}\left(x_{i-1}, x_{i}\right),
$$

where $\phi_{i}$ is defined as

$$
\begin{aligned}
\phi_{i}\left(x_{i-1}, x_{i}\right) & =\alpha \times d\left(g_{i}^{*}, g_{i}\right)^{2} \\
& +\beta \times\left(A_{\overline{\mathcal{S}}^{2}}\left(p_{i-1}^{*}, p_{i}^{*}\right)-A_{\overline{\mathcal{S}}^{2}}\left(x_{i-1}, x_{i}\right)\right),
\end{aligned}
$$

where $x_{i-1}$ and $x_{i}$ are sampled points of $\overline{\mathcal{S}}^{2}$ using a candidate sampler for the $(i-1)^{t h}$ and $i^{t h}$ position, such that $g_{i} x_{i-1}=x_{i}$. Moreover, only the penalty term of $\overline{\mathcal{S}}^{2}$ is added in (32), since the sampling of $\mathcal{S}_{*}^{1}$ is fixed to uniform sampling its penalty is zero. Subsequently, (32) is identified as the composition of linearly sequential sub-problems. As a result, its solution is estimated by computing the minimum and the minimizer of (32) sequentially, see Appendix. Assuming the same search space size, i.e. $\forall i, s=\left|U_{i}\right|$, the optimal sampling function can be estimated in $\mathcal{O}\left(s^{2} z\right)$ time for open curves. The point correspondence estimation, in closed 

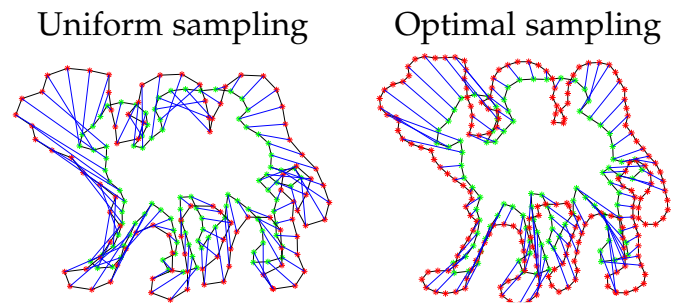

Fig. 9: Optimal vs Uniform sampling. For the optimal case, the green curves are sampled uniformly, and the curves in red optimally. Note that the optimal sampler adjusts the sampling rate while the uniform sampling does not.

curves, follows the same procedure. However, unlike the open curves the starting point is not known a priori. Hence, the optimal sampling for every point as a starting point is computed and the point with the least cost will be chosen as a starting point along with its sampling solution.

\subsection{Uniform sampling}

In this subsection, we will present point correspondence estimation for uniformly sampled shapes, as discussed in [15]. The approach restricts shape sampling functions to a group of $z$-cyclic permutations, given the shapes to be aligned are uniformly sampled. To elaborate, given two uniformly sampled closed curves $\mathcal{S}_{*}^{1}$ and $\mathcal{S}_{*}^{2}$ the estimation of point correspondence is formulated as estimating the starting point and the orientation of $\mathcal{S}_{*}^{2}$; since the sampling rate is uniform it is not optimized. Subsequently, a family of uniform samplers is defined as: $\xi_{*}^{i}:[0, z] \ni j \rightarrow(i+j) \bmod z \in[0, z]$, where mod represents the modulo operation. As a result, for a fixed orientation the optimal staring point is estimated by running through all possible starting points and selecting the one that minimizes the following

$$
\mathcal{I}\left(f\left(\mathcal{S}_{*}^{1}\right), f\left(\mathcal{S}_{*}^{2}\right)\right)=\min _{i \in[1, z]} \mathfrak{d}\left(f\left(\mathcal{S}_{*}^{1}\right), f\left(\mathcal{S}_{*}^{2} \circ \xi_{*}^{i}\right)\right) .
$$

Note that (34) is a restricted version of (30). Furthermore, the approach considers orientation reversing matchings by generalizing (34) to the following objective functional

$$
\arg \min \left(\mathcal{I}\left(f\left(\mathcal{S}_{*}^{1}\right), f\left(\overrightarrow{\mathcal{S}_{*}^{2}}\right)\right), \mathcal{I}\left(f\left(\mathcal{S}_{*}^{1}\right), f\left(\overleftarrow{\mathcal{S}_{*}^{2}}\right)\right)\right)
$$

where $\rightarrow$ and $\leftarrow$, represent ordering in clock-wise and anti-clockwise direction, respectively. Meanwhile, the point correspondence between two given open curves is done by testing only the different end points as a starting point. The solution of (35) is estimated iteratively using nested loops. Thus, the time-complexity of aligning closed curves is $\mathcal{O}\left(z^{2}\right)$. Moreover, it is worth to note that if $f\left(\overrightarrow{\mathcal{S}_{*}^{2}}\right)=\left(g_{1}, \cdots, g_{z}\right)$ then $f\left(\overleftarrow{\mathcal{S}_{*}^{2}}\right)=\left(g_{z}^{-1}, \cdots, g_{1}^{-1}\right)$. In comparison, if optimal sampling is used for estimating the point correspondence between closed shapes the time-complexity would be $\mathcal{O}\left(s^{2} z^{2}\right)$. Although computationally efficient, the uniform sampling gives a meaningful matching only for shapes with small deformations or linearly elastic deformations. On the contrary, the optimal sampling scheme gives consistently meaningful matching even under occlusion and missing parts, see Fig. 9.

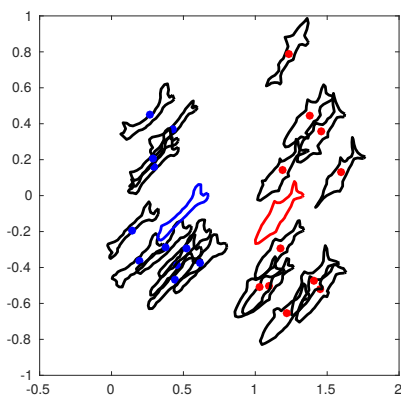

(a)

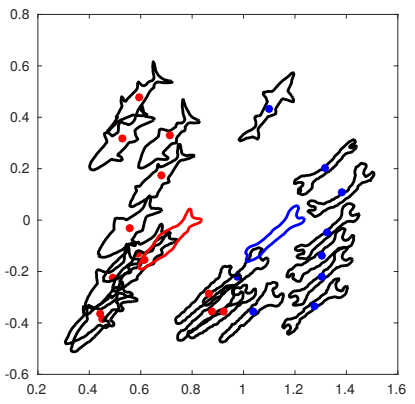

(b)
Fig. 10: Examples of clustering results for $\mathrm{k}=2$. (a) based on optimal sampling. (b) based on uniform sampling.

\section{Application in SHAPE ANALYSIS}

As discussed in earlier sections, the proposed representation space, $\operatorname{SE}(\mathrm{n})^{z}$, is a curved space. Nevertheless, it is possible to fit statistical models and do further shape analysis using the defined distance metric, (20). Particularly, in [14] an inhomogeneous time Markov process is used to capture the statistical properties of a deformable shape represented in a matrix Lie group. Grouping a collection of data into distinct clusters is an alternative data analysis technique that is extensively studied in a wide range of problems. Apart from the well-known k-means algorithm, there are several clustering algorithms emphasizing different aspects of the problem [46], [59]. However, the performance of a clustering algorithm depends on a goal specific similarity metric, among other factors. In that regard, we propose (20) or (35), depending on the assumption, as a similarity metric to cluster shapes. To that end, we discuss modelling of a shape class, represented in $\mathrm{SE}(\mathrm{n})^{z}$, by k-means clustering.

\subsection{K-means for shape clustering}

In k-means clustering, the central idea is to estimate $\mathrm{k}$ points such that the data points around these $\mathrm{k}$ centroids are more similar with each other under the metric being considered. Moreover, the $\mathrm{k}$ points are thought of as the means of the clusters [35]. Consequently, we will mainly discuss the computation of arithmetic mean in $\mathrm{SE}(\mathrm{n})^{z}$. Over the past years, considerable work has been done on characterizing the mean of several matrices [4], [9], [25]. For our purpose, however, we will consider Fréchet mean and its local construction called Karcher mean [26], [47]. Fréchet mean is a generalization of a distribution's centroid in any metric space $\mathcal{U}$; it is defined as

$$
\min _{\tilde{x} \in \mathcal{U}} \sum_{y \in \mathcal{U}} \delta(\tilde{x}, y)^{2},
$$

where $\delta(\cdot, \cdot)$ denotes a distance metric. Essentially, it characterizes the mean of a distribution, under a given metric, as a value that minimizes the dispersion of the dataset. Subsequently, by replacing the distance metric $\delta(\cdot, \cdot)$ with (18) we get the following objective function for computing the mean of $m$ rigid transformation matrices.

$$
\min _{\tilde{R} \in \mathrm{SO}(\mathrm{n})} \sum_{j=1}^{m}\left\|\log \left(\tilde{R}^{T} R_{j}\right)\right\|_{F}^{2}+\min _{\tilde{v} \in \mathbb{R}^{n}} \sum_{j=1}^{m}\left\|v_{j}-\tilde{v}\right\|_{2}^{2} .
$$


The second term of (37) is satisfied by taking the arithmetic mean, since $\mathbb{R}^{n}$ is a vector space. For the first term, however, an optimal global solution is not ensured. Thus, the local solution of Fréchet mean (Karcher mean) is computed [1], [47] - a direct product of (37) is optimized to compute the mean of shape representations. Subsequently, given a prior knowledge of $k$, expectation-maximization (EM) algorithm is used to estimate the $\mathrm{k}$ mean points and the clusters around them, see Fig. 10. Note that, results of the EM algorithm depend on the initialization. If the $\mathrm{k}$ is not known a priori alternative assumptions can be made on the distribution of the data to automatically estimate $\mathrm{k}$ [24]. In general, k-means of shape clusters are estimated by optimizing the following with EMalgorithm

$$
\min \sum_{j=1}^{m} \sum_{i=1}^{k} r_{i j} \mathfrak{d}\left(f\left(\tilde{\mathcal{S}}^{i}\right), f\left(\mathcal{S}^{j}\right)\right)^{2},
$$

where $f\left(\tilde{\mathcal{S}}_{i}\right)$ is the representation of the estimated mean shape of the $i^{\text {th }}$ cluster, and $r_{i j}$ is 1 if and only if the $j^{\text {th }}$ shape is assigned to the $i^{\text {th }}$ cluster otherwise is 0 .

\section{EXPERIMENTAL RESULTS}

In this section, we present two main experimental scenarios and a concrete computational time cost of the approach. In Subsection 7.1, an evaluation of the accuracy and representativeness of the proposed approach is presented. In Subsection 7.2, the impact of occlusion, sampling point variation, and local perturbation on the proposed approach is presented. In Subsection 7.3, the computational cost of our approach and [54] is presented.

\subsection{Shape retrieval}

Given a query shape, a shape retrieval system tries to rank shapes in a given database according to their similarity to the query. Usually, such systems are solely based on a distance metric rather than a mathematical model of shape categories. Hence, the performance of a shape retrieval system highlights the representativeness of the used distance metric. As a result, we present the evaluation of a shape retrieval system that is based on the proposed distance metric (34) and (32) on different datasets.

Flavia leaf dataset: The dataset contains 32 types of leaf species with a total of 1907 examples see Fig. 11a. In [31] a leave-one-out test scenario was performed on the dataset to evaluate an elastic similarity metric derived from SRVframework [54]. Leave-one-out is a setup where every leaf is used as a query against the rest of the dataset. To compare our approach with other methods, we also replicate the leave-one-out scenario with Mean Average Precision (MAP) used as a performance measure. For this experiment, every leaf shape is represented by $z=200$ points that are uniformly sampled from its boundary. Table 3 summarizes the result of our approach and results reported in [31] and [43]. Although our method achieved high MAP, it is not necessarily inclusive of all relevant informations; precision drops as recall goes to 1, see Fig. 12a. Nonetheless, it outperformed the elastic shape metric and the Gaussian elastic metric, discussed in [31], in terms of MAP. One possible reason for this is that we do not pre-smooth the data and thus local details are more likely to be captured with our method.

Swedish leaf: The Swedish leaf dataset contains 1125 example leaves from 15 leaf types. The example shapes are distributed uniformly; there are 75 example leaves from each species. To compare our approach with other methods, we follow the same experimental scenario discussed in [31], [43], which is nearest neighbour classification. We randomly select 25 leaf shapes from each type and use the left out 50 for testing. We repeat the experiment 100 times and take the average classification rate. In all of the 100 experiments, the shapes are represented by $z=200$ uniformly sampled points. Our method achieved an average classification rate of 99.50 with 0.01287 standard deviation. Table. 4 shows the comparison of our result with other shape matching methods. We note that performance of a nearest neighbour classifier is not an explicit measure of a similarity metric's performance, as the classification is based on the best result among elements of a group. Consequently, in Fig. 12a we show the precision-recall (PR) curve of a retrieval result obtained by performing the leave-one-out experimental scenario on the dataset. Note that, the area under the PR curve is smaller in the Swedish leaf case as compared to the area under the PR curve for the Flavia leaf dataset. In general, we observe that the inter-class similarity in the Flavia leaf dataset is high as compared to the Swedish leaf dataset making discrimination in Flavia difficult, see Fig. 11. However, the intra-class variation in the Swedish dataset is mainly due to nonlinear elastic deformations, whereas in the Flavia it is mainly due to a combination of rotation and scaling. Hence, distance metric based on uniform sampling, see Subsection 5.2, does not perform well in the Swedish leaf dataset as compared to the Flavia leaf dataset, in terms of precision and recall.

Generic shapes: To evaluate the impact of optimal sampling based point correspondence estimation, we tested our approach on generic shape datasets, MPEG-7 [32], Kimia99 and Kimia216 [51]. The MPEG-7 dataset contains 70 shape classes each containing 20 elements, Kimia99 is composed of 9 shape classes with 11 examples each, and Kimia216 contains 18 class of shapes with 12 examples. All the datasets are composed of shape categories with variations due to different view point and/or large deformations. As a result, these datasets pose a significantly challenging problem than either the Flavia or the Swedish leaf dataset do. We evaluate our approach, on all datasets, by replicating the experimental scenario described in [5] and [58], which is similar to the leave-one-out. In the case of Kimia99 and Kimia216 retrieval accuracy is measured by counting the overall results of the top 10 (in Kimia99) and top 11 (in Kimia216) retrievals from the same class, excluding the query shape [58]. Meanwhile, retrieval accuracy on the MPEG-7 dataset is measured based on what is called "Bull's eye score"- Bull's eye score takes the overall percentage of retrieval results, among the first 40 , that belong to the same class as the query [58]. In all datasets, we evaluate our approach for $z=100$ using both uniform and optimal sampling-for the optimal case the query shape is sampled uniformly and each element from the dataset are sampled optimally using (32). More importantly, in both cases, matching starting points and shape orientation are estimated as discussed in Section 5.2 and 3, respectively. Furthermore, $\beta=0.1, \alpha=1$, and 


\begin{tabular}{|c|cccccccccc|}
\hline Methods & 1st & 2nd & 3rd & 4th & 5th & 6th & 7th & 8th & 9th & 10th \\
\hline SC [7] & 97 & 91 & 88 & 85 & 84 & 77 & 75 & 66 & 56 & 37 \\
Shock graph [51] & 99 & 99 & 99 & 98 & 98 & 97 & 96 & 95 & 93 & 82 \\
Height functions [58] & 99 & 99 & 99 & 99 & 98 & 99 & 99 & 96 & 95 & 88 \\
Symbolic representation [13] & 99 & 99 & 99 & 98 & 99 & 98 & 98 & 95 & 96 & 94 \\
Our method with optimal sampling & 99 & 99 & 97 & 97 & 97 & 98 & 93 & 90 & 79 & 53 \\
Our method with uniform sampling & 91 & 81 & 73 & 75 & 63 & 57 & 51 & 44 & 35 & 30 \\
\hline
\end{tabular}

TABLE 1: Retrieval results on Kimia99 shape dataset.

\begin{tabular}{|c|ccccccccccc|}
\hline Methods & 1st & 2nd & 3rd & 4th & 5th & 6th & 7th & 8th & 9th & 10th & 11th \\
\hline SC [7] & 214 & 209 & 205 & 197 & 191 & 178 & 161 & 144 & 131 & 101 & 78 \\
Shock graph [51] & 216 & 216 & 216 & 215 & 210 & 210 & 207 & 204 & 200 & 187 & 163 \\
Skeleton graph matching [5] & 216 & 216 & 215 & 216 & 213 & 210 & 210 & 207 & 205 & 191 & 177 \\
Our method with optimal sampling & 216 & 216 & 208 & 205 & 203 & 193 & 192 & 178 & 169 & 162 & 123 \\
Our method with uniform sampling & 212 & 210 & 188 & 181 & 174 & 165 & 159 & 151 & 141 & 132 & 120 \\
\hline
\end{tabular}

TABLE 2: Retrieval results on Kimia216 shape dataset.

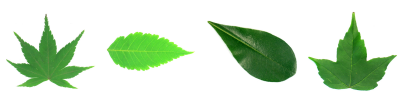

(a)

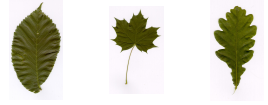

(b)

Fig. 11: Examples of different leaf types. (a) from the Flavia leaf dataset. (b) from the Swedish leaf dataset.

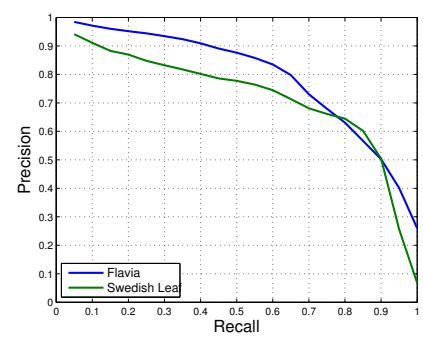

(a)

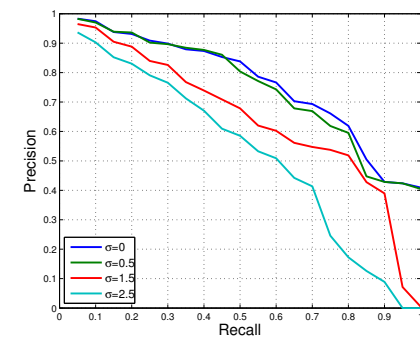

(b)

Fig. 12: (a) PR curves on the Flavia and the Swedish leaf dataset. (b) PR curves under different noise magnitudes introduced to the original fighter jet planes dataset.

$\eta=20$ in all of the optimal sampling based point matchings. In Table. 1 and 2 we compare our result with other approaches evaluated on Kimia99 and Kimia216. On MPEG-7, retrieval based on the uniform sampling approach achieved a bull's eye score of $68.02 \%$, while retrieval based on the optimal sampling approach achieved $84.17 \%$. We note that retrieval results based on optimal samplings achieve a better score than results based on uniform samplings, in all three datasets. In general, given a large $\eta$ and a small $\beta$ (relative to $\alpha$ ) retrieval based on an optimal sampling should perform better than retrieval based on a uniform sampling. Nevertheless, we stress that the reported optimal sampling based results are based on estimates of the starting point and the shape orientation alignment from the uniform sampling approach. Hence, the optimal sampling inherits misalignment errors from the uniform sampling, especially in cases of large deformations, which lead to performance degradation.

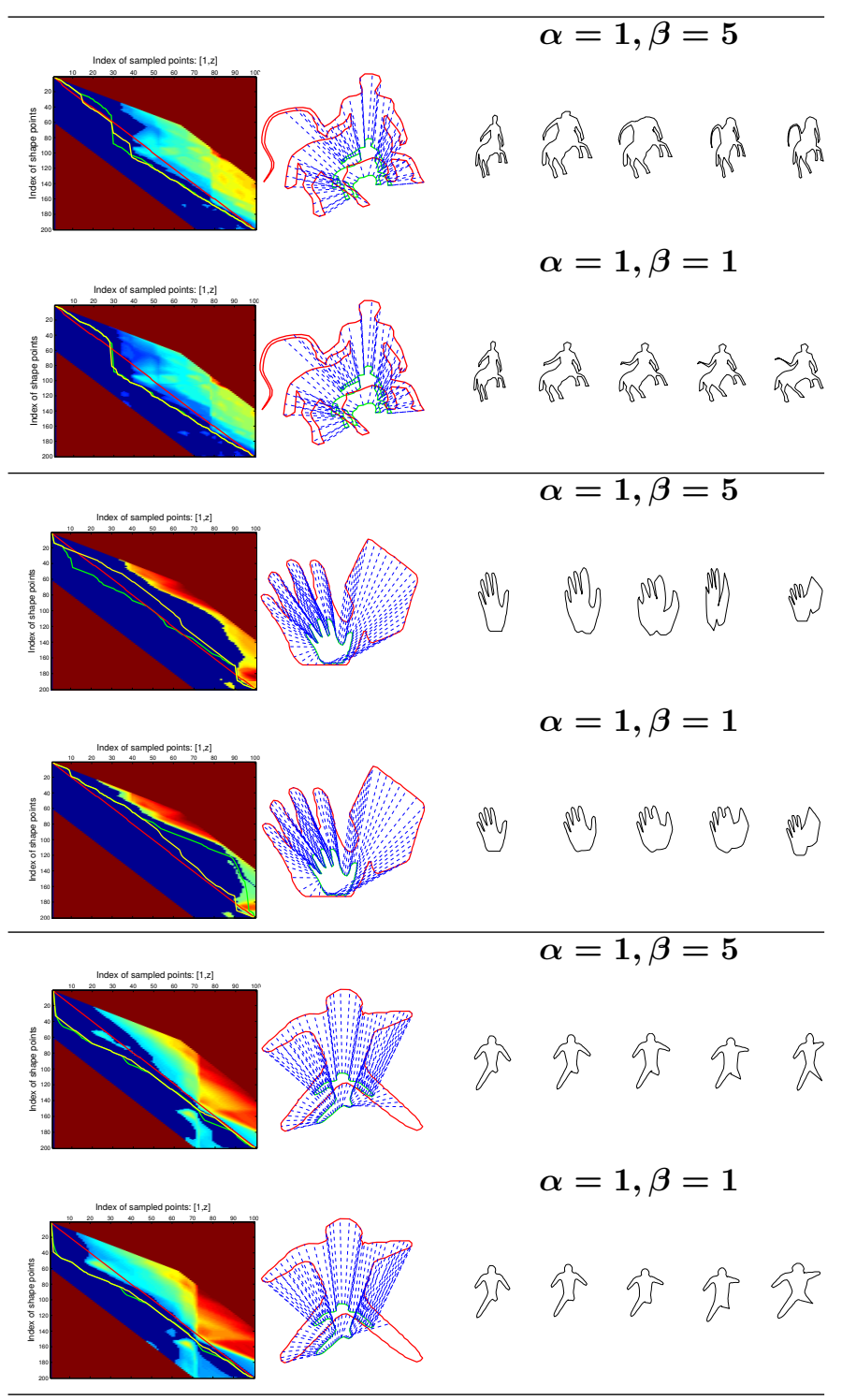

Fig. 13: Impact of $\beta$ on partial shape matching. In all of the above examples, $\eta=37.46$. Each row shows optimal sampling of a shape, shown in red, to match the uniformly sampled shape shown in green. Note that, a large value of $\beta$ leads to a matching that favours area preservation. 


\begin{tabular}{|l|l|}
\hline Methods & MAP \\
\hline Angle function [30] & 45.87 \\
Shape context [7] & 47.00 \\
TSLA [43] & 69.93 \\
Elastic metric with 200 points [31] & 81.86 \\
Gaussian elastic metric with 200 points [31] & 92.37 \\
Our method with 200 points & $\mathbf{9 4 . 1 1}$ \\
\hline
\end{tabular}

TABLE 3: MAP on the Flavia leaf dataset. Our result is highlighted at the bottom.

\begin{tabular}{|l|c|}
\hline Methods & Recognition rate \\
\hline Fourier descriptors [33] & 89.60 \\
Shape-Tree [18] & 96.28 \\
TSLA [43] & 96.53 \\
Elastic metric with 200 points [31] & 99.18 \\
Our approach with 200 points & $\mathbf{9 9 . 5 0}$ \\
\hline
\end{tabular}

TABLE 4: Nearest-neighbour recognition rate on the Swedish leaf dataset. Our result is highlighted at the bottom.

\subsection{Robustness}

In this subsection, we test the robustness of the proposed similarity metric to local noise and sampling size variations, and the effectiveness of optimal sampling under occlusion. To that end, we use shapes from fighter jets dataset [56], Mythological creatures [11], and 1070-Shape dataset that can be found at http://vision.lems.brown.edu/content/ available-software-and-databases.

Local shape perturbations: In general, local perturbation of a curved shape with a noise that does not alter the shape degrades the performance of a shape alignment and retrieval system. Consequently, we evaluate the tolerance of the proposed approach to local perturbations on fighter jets dataset [56]. The dataset contains 7 types of fighter jets each with 30 examples. The main cause of intra-class variation is deforming parts of the plane and rotation. We begin our experiment by introducing an additive white Gaussian noise to the shapes of the fighter jets. We denote the standard deviation of the noise by $\sigma$. For all subsequent experiments, the contour of each shape is approximated by uniformly sampled $z=200$ points. Next, we do a leave-one-out test scenario where the original (noise free) dataset is queried by every shape from datasets corrupted by noise with different distributions. Table. 5 summarizes the computed MAP values and Fig. 12b shows their respective precisionrecall curve. In general, the proposed similarity metric is tolerant to local perturbations that do not alter the shape significantly.

Correspondence estimation under occlusion: Shapes can exhibit partial similarity due to occlusions or nonlinear elastic deformations, see Fig. 13. Thus, a point correspondence estimation method has to be able to handle nonlinear elastic deformations. For instance, the uniform sampling, discussed in Subsection 5.2, is constrained to linear elastic deformations, thus it fails to match shapes that are partially similar, see Fig. 9. Alternatively, the optimal sampling, presented in

\begin{tabular}{|c|c|c|c|c|}
\hline$\sigma$ & 0 & 0.5 & 1.5 & 2.5 \\
\hline MAP & 97.11 & 96.72 & 89.95 & 83.27 \\
\hline
\end{tabular}

TABLE 5: MAP on the fighter jets dataset with a Gaussian noise of different standard deviations.

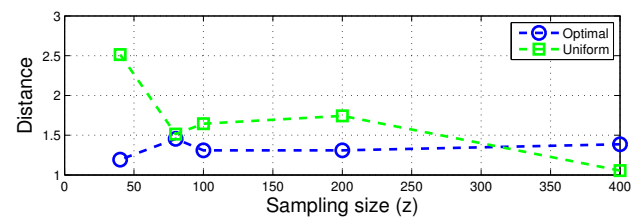

Fig. 14: Impact of sampling size under the optimal and the uniform sampling based point correspondence estimation.

Subsection 5.1, can be tuned to perform well by adjusting $\beta$ in (32). To illustrate this, we select example shapes with partial similarity from Mythological creatures and 1070-shape datasets. Subsequently, in Fig. 13 we show matching results for different values of $\beta$. In all the matching scenarios, the elasticity constraint $\eta$ is held constant to 37.46 . Hence, the solution search space is fixed. Nevertheless, in case of occlusion or partial matching, we note that forcing the sampler to preserve area when that is clearly not the case leads to point mismatching, hence, a small value should be assigned to $\beta$.

Effects of sampling size: The proposed approach casts the point correspondence estimation problem as a sampling function estimation problem, hence the sampling function is optimized and not given. However, the number of points $z$ is selected a priori. Here, we investigate the impact of having different values of $z$ on the optimal point correspondence estimation and its accuracy. In general approximating a shape, especially one with intricate structure, by a small number of points leads to a less detailed result. Nevertheless, it is desirable to have a consistent distance for different sample sizes. To that end, Fig. 14 shows how the distance between two open curves, shown in Fig. 15, varies under different sampling sizes for both the uniform and the optimal sampling. The result is obtained by varying $z$ while the rest of the parameters are fixed $\alpha=1, \beta=10$. We observe that the distance under the optimal sampling is more consistent relative to the uniform sampling based distance.

\subsection{Computational cost}

As discussed earlier, the geodesic distance between two given curves can be computed explicitly. Hence, the computational cost of a geodesic distance is completely dependent on the computational cost of the point correspondence estimation approaches, which are $\mathcal{O}\left(s^{2} z^{2}\right)$ and $\mathcal{O}\left(z^{2}\right)$ for the optimal and the uniform sampling, respectively. The entire proposed approach, from the computation of the representation matrices up to the optimal sampling estimation, is implemented in MATLAB R2014a running on an Intel core $17-3540 \mathrm{M}$ with $3.0 \mathrm{GHz} \times 4$ processing speed and 7.7 GB RAM with Ubuntu 14.04 64-bit operating system. Henceforth, the reported computation cost is for shapes approximated by $z=100$ sampled points and includes the cost of computing the representation $f(\cdot)$ and the preprocessing stage. Subsequently, the geodesic distance (20) computation, assuming established point correspondence, takes 0.1045 seconds. Point correspondence estimation, under the uniform sampling, between two closed curves, shown in Fig. 2 first row, takes 11.1346 seconds. The same 

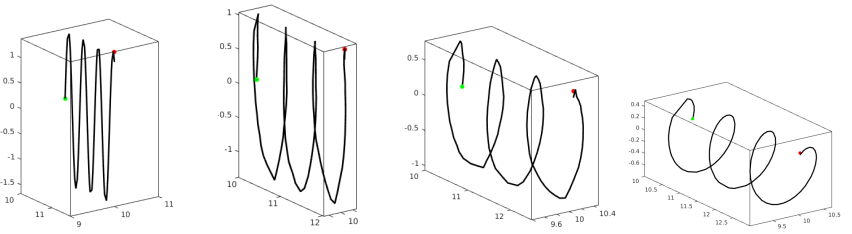

Fig. 15: A geodesic path from the first to the last curve. These curves are used in estimating the effects of sample size.

matching problem is estimated with a $\mathrm{C}++$ implementation of SRV framework [54] running on the same machine in 2.0298 seconds. Meanwhile, the optimal sampling of open curves or closed curves with a known starting point and orientation has taken 64.5833 seconds for $\eta=7.013$. In general, the computational cost of the optimal sampling is much higher than both SRV and the uniform sampling.

\section{CONCLUSION}

We have presented a deformation based representation approach for curved shapes. The approach considers curves to be elements of a finite dimensional matrix Lie group. Such a representation led to an explicit geodesic equation and a left invariant distance metric. As a result, the geodesic curve and distance between shapes can be computed in a closed form and statistical analysis of shapes can be done in a finite dimensional matrix space. Furthermore, there is less restriction on the family of curves being considered as compared to other parametrization approaches; ours does not require curves to be differentiable as long as there is continuity in a topological sense. However, curves with changing topology are beyond the scope of the representation. Moreover, two possible solutions for point correspondence estimation are presented. In both solutions, the problem of point correspondence is treated as a sampling function estimation problem. Although computationally expensive, the optimal sampling estimation provides an explicit control over elasticity constraint through weighting constants and window sizes. Experimental results show that the approach gives a discriminative representation that is robust to local perturbation, displacement and occlusion. Mainly, in comparison to the SRV framework the MATLAB implementation of the approach is slower.

\section{REFERENCES}

[1] P-A Absil, Robert Mahony, and Rodolphe Sepulchre. Optimization algorithms on matrix manifolds. Princeton University Press, 2009. 10

[2] Yali Amit. A nonlinear variational problem for image matching. SIAM Journal on Scientific Computing, 15(1):207-224, 1994. 6

[3] Yali Amit, Ulf Grenander, and Mauro Piccioni. Structural image restoration through deformable templates. Journal of the American Statistical Association, 86(414):376-387, 1991. 3

[4] T Ando, Chi-Kwong Li, and Roy Mathias. Geometric means. Linear algebra and its applications, 385:305-334, 2004. 9

[5] Xiang Bai and Longin Jan Latecki. Path similarity skeleton graph matching. IEEE transactions on pattern analysis and machine intelligence, 30(7):1282-1292, 2008. 10, 11

[6] Richard E Bellman and Stuart E Dreyfus. Applied dynamic programming. Princeton university press, 2015. 7
[7] Serge Belongie, Jitendra Malik, and Jan Puzicha. Shape matching and object recognition using shape contexts. Pattern Analysis and Machine Intelligence, IEEE Transactions on, 24(4):509-522, 2002. 1, 11,12

[8] Rajendra Bhatia. Positive definite matrices. Princeton University Press, 2009. 5

[9] Dario Bini, Beatrice Meini, and Federico Poloni. An effective matrix geometric mean satisfying the ando-li-mathias properties. Mathematics of Computation, 79(269):437-452, 2010. 9

[10] Fred L Bookstein. Size and shape spaces for landmark data in two dimensions. Statistical Science, pages 181-222, 1986. 2

[11] Alexander M Bronstein, Michael M Bronstein, Alfred M Bruckstein, and Ron Kimmel. Analysis of two-dimensional non-rigid shapes. International Journal of Computer Vision, 78(1):67-88, 2008. 12

[12] Isaac Cohen, Nicholas Ayache, and Patrick Sulger. Tracking points on deformable objects using curvature information. In Computer VisionECCV'92, pages 458-466. Springer, 1992. 7

[13] Mohammad Reza Daliri and Vincent Torre. Robust symbolic representation for shape recognition and retrieval. Pattern Recognition, 41(5):1782-1798, 2008. 11

[14] Girum Demisse, Djamila Aouada, and Björn Ottersten. Templatebased statistical shape modelling on deformation space. In 22nd IEEE International Conference on Image Processing, 2015. 2, 9

[15] Girum Demisse, Djamila Aouada, and Björn Ottersten. Similarity metric for curved shapes in euclidean space. In The IEEE Conference on Computer Vision and Pattern Recognition (CVPR), June 2016. 1, 2, 5,9

[16] Manfredo Perdigao do Carmo Valero. Riemannian geometry. 1992. 4,5

[17] Ian L Dryden and Kanti V Mardia. Statistical shape analysis, volume 4. Wiley Chichester, 1998. 2

[18] Pedro F Felzenszwalb and Joshua D Schwartz. Hierarchical matching of deformable shapes. In Computer Vision and Pattern Recognition, 2007. CVPR'07. IEEE Conference on, pages 1-8. IEEE, 2007. 12

[19] Oren Freifeld and Michael J Black. Lie bodies: A manifold representation of 3d human shape. In Computer Vision-ECCV 2012, pages 1-14. Springer, 2012. 1, 2, 3, 6

[20] Ulf Grenander. Geometries of knowledge. Proceedings of the National Academy of Sciences, 94(3):783-789, 1997. 3

[21] Ulf Grenander and Daniel Macrae Keenan. On the shape of plane images. SIAM Journal on Applied Mathematics, 53(4):1072-1094, 1993. 2,3

[22] Ulf Grenander and Michael I Miller. Representations of knowledge in complex systems. Journal of the Royal Statistical Society. Series B (Methodological), pages 549-603, 1994. 2, 3

[23] Ulf Grenander and Michael I Miller. Pattern theory: from representation to inference. Oxford University Press, 2007. 3

[24] Greg Hamerly and Charles Elkan. Learning the k in k means. Advances in neural information processing systems, 16:281, 2004. 10

[25] Jeffrey Ho, Guang Cheng, Hesamoddin Salehian, and Baba Vemuri. Recursive karcher expectation estimators and geometric law of large numbers. In Proceedings of the Sixteenth International Conference on Artificial Intelligence and Statistics, pages 325-332, 2013. 9

[26] Hermann Karcher. Riemannian center of mass and mollifier smoothing. Communications on pure and applied mathematics, 30(5):509-541, 1977. 9

[27] Michael Kass, Andrew Witkin, and Demetri Terzopoulos. Snakes: Active contour models. International journal of computer vision, 1(4):321-331, 1988

[28] David G Kendall. Shape manifolds, procrustean metrics, and complex projective spaces. Bulletin of the London Mathematical Society, 16(2):81-121, 1984. 2, 3

[29] N Khaneja, Michael I. Miller, and Ulf Grenander. Dynamic programming generation of curves on brain surfaces. IEEE Transactions on Pattern Analysis and Machine Intelligence, 20(11):1260-1265, 1998. 3

[30] Eric Klassen, Anuj Srivastava, Washington Mio, and Shantanu H Joshi. Analysis of planar shapes using geodesic paths on shape spaces. Pattern Analysis and Machine Intelligence, IEEE Transactions on, 26(3):372-383, 2004. 1, 2, 12

[31] Hamid Laga, Sebastian Kurtek, Anuj Srivastava, and Stanley J Miklavcic. Landmark-free statistical analysis of the shape of plant leaves. Journal of theoretical biology, 363:41-52, 2014. 10, 12 
[32] Longin Jan Latecki, Rolf Lakamper, and T Eckhardt. Shape descriptors for non-rigid shapes with a single closed contour. In Computer Vision and Pattern Recognition, 2000. Proceedings. IEEE Conference on, volume 1, pages 424-429. IEEE, 2000. 10

[33] Haibin Ling and David W Jacobs. Shape classification using the inner-distance. Pattern Analysis and Machine Intelligence, IEEE Transactions on, 29(2):286-299, 2007. 12

[34] Wei Liu, Anuj Srivastava, and Jinfeng Zhang. Protein structure alignment using elastic shape analysis. In Proceedings of the First ACM International Conference on Bioinformatics and Computational Biology, pages 62-70. ACM, 2010. 1

[35] James MacQueen et al. Some methods for classification and analysis of multivariate observations. In Proceedings of the fifth Berkeley symposium on mathematical statistics and probability, volume 1, pages 281-297. Oakland, CA, USA., 1967. 9

[36] Siddharth Manay, Daniel Cremers, Byung-Woo Hong, Anthony J Yezzi, and Stefano Soatto. Integral invariants for shape matching. Pattern Analysis and Machine Intelligence, IEEE Transactions on, 28(10):1602-1618, 2006. 3, 5

[37] Andrea CG Mennucci. Metrics of curves in shape optimization and analysis. In Level Set and PDE Based Reconstruction Methods in Imaging, pages 205-319. Springer, 2013. 1, 2, 3, 5

[38] Peter W Michor and David Mumford. Riemannian geometries on spaces of plane curves. arXiv preprint math/0312384, 2003. 1, 2

[39] Peter W Michor and David Mumford. An overview of the riemannian metrics on spaces of curves using the hamiltonian approach Applied and Computational Harmonic Analysis, 23(1):74-113, 2007. 1

[40] John Milnor. Curvatures of left invariant metrics on lie groups. Advances in mathematics, 21(3):293-329, 1976. 5

[41] Maher Moakher. Means and averaging in the group of rotations. SIAM journal on matrix analysis and applications, 24(1):1-16, 2002. 5

[42] Frank Morgan and James F Bredt. Riemannian geometry: a beginner's guide. Jones and Bartlett, 1993. 4

[43] Sofiene Mouine, Itheri Yahiaoui, and Anne Verroust-Blondet. A shape-based approach for leaf classification using multiscaletriangular representation. In Proceedings of the $3 r d$ ACM conference on International conference on multimedia retrieval, pages 127-134. ACM, 2013. 10,12

[44] David Mumford. Pattern theory: a unifying perspective. In First European congress of mathematics, pages 187-224. Springer, 1994. 1

[45] David Mumford and Agnès Desolneux. Pattern theory: the stochastic analysis of real-world signals. CRC Press, 2010. 6, 7

[46] Andrew Y Ng, Michael I Jordan, Yair Weiss, et al. On spectral clustering: Analysis and an algorithm. Advances in neural information processing systems, 2:849-856, 2002. 9

[47] Xavier Pennec. Intrinsic statistics on riemannian manifolds: Basic tools for geometric measurements. Journal of Mathematical Imaging and Vision, 25(1):127-154, 2006. 9, 10

[48] Xavier Pennec. Statistical computing on manifolds: from riemannian geometry to computational anatomy. In Emerging Trends in Visual Computing, pages 347-386. Springer, 2009. 1

[49] Clayton Scott and Robert Nowak. Robust contour matching via the order-preserving assignment problem. IEEE Transactions on Image Processing, 15(7):1831-1838, 2006. 7

[50] Thomas B Sebastian, Philip N Klein, and Benjamin B Kimia. On aligning curves. Pattern Analysis and Machine Intelligence, IEEE Transactions on, 25(1):116-125, 2003. 7

[51] Thomas B Sebastian, Philip N Klein, and Benjamin B Kimia Recognition of shapes by editing their shock graphs. IEEE Transactions on pattern analysis and machine intelligence, 26(5):550-571, 2004. 10,11

[52] Eitan Sharon and David Mumford. 2d-shape analysis using conformal mapping. International Journal of Computer Vision, 70(1):5575, 2006. 1

[53] Christopher G Small. The Statistical Theory of Shape, Springer Series in Statistics. New York: Springer-Verlag, 1996. 2, 3

[54] Anuj Srivastava, Eric Klassen, Shantanu H Joshi, and Ian $\mathrm{H}$ Jermyn. Shape analysis of elastic curves in euclidean spaces. Pattern Analysis and Machine Intelligence, IEEE Transactions on, 33(7):1415-1428, 2011. 2, 3, 5, 6, 10, 13

[55] Hemant D Tagare. Shape-based nonrigid correspondence with application to heart motion analysis. Medical Imaging, IEEE Transactions on, 18(7):570-579, 1999. 7

[56] Ninad Thakoor, Jean Gao, and Sungyong Jung. Hidden markov model-based weighted likelihood discriminant for 2-d shape classification. Image Processing, IEEE Transactions on, 16(11):2707-2719, 2007. 12
[57] Alain Trouvé. An infinite dimensional group approach for physics based models in pattern recognition. preprint, 1995. 3, 6

[58] Junwei Wang, Xiang Bai, Xinge You, Wenyu Liu, and Longin Jan Latecki. Shape matching and classification using height functions. Pattern Recognition Letters, 33(2):134-143, 2012. 10, 11

[59] Mingrui Wu and Bernhard Schölkopf. A local learning approach for clustering. In Advances in neural information processing systems, pages 1529-1536, 2006. 9

[60] Mingqiang Yang, Kidiyo Kpalma, Joseph Ronsin, et al. A survey of shape feature extraction techniques. Pattern recognition, pages 43-90, 2008. 1

[61] Laurent Younes. Computable elastic distances between shapes. SIAM Journal on Applied Mathematics, 58(2):565-586, 1998. 2, 3, 6, 7

[62] Laurent Younes. Optimal matching between shapes via elastic deformations. Image and Vision Computing, 17(5):381-389, 1999. 3

[63] Laurent Younes. Parametrized Plane Curves. Springer, 2010. 1, 3, 6, 7

[64] Laurent Younes. Spaces and manifolds of shapes in computer vision: An overview. Image and Vision Computing, 30(6):389-397, 2012. 1, 2

[65] Milos Zefran, Vijay Kumar, and Christopher B Croke. On the generation of smooth three-dimensional rigid body motions. Robotics and Automation, IEEE Transactions on, 14(4):576-589, 1998. 5

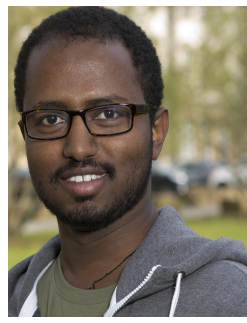

Girum G. Demisse received Bsc in computer science from Hawassa University, Ethiopia, and Msc in computer science-smart systems -from Jacobs University Bremen, Germany, in 2008 and 2013 , respectively. He is currently studying for a $\mathrm{PhD}$ degree in computer science at the Interdisciplinary Center for Security, Reliability, and Trust, University of Luxembourg. His main research interests lie at the intersection of mathematical modelling, computer vision and machine learning.

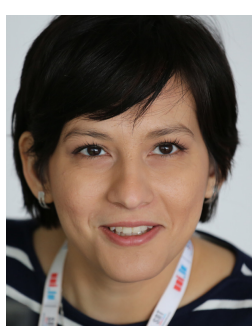

Djamila Aouada (S'05-M'09) received the State Engineering degree in electronics in 2005, from the École Nationale Polytechnique (ENP), Algiers, Algeria, and the Ph.D. degree in electrical engineering in 2009 from North Carolina State University (NCSU), Raleigh, NC. She is Research Scientist at the Interdisciplinary Centre for Security, Reliability, and Trust (SnT), at the University of Luxembourg. Dr. Aouada has been leading the computer vision activities at the SnT since 2009. She has worked as a consultant for multiple renowned laboratories (Los Alamos National Laboratory, Alcatel Lucent Bell Labs., and Mitsubishi Electric Research Labs.). Her research interests span the areas of signal and image processing, computer vision, pattern recognition and data modelling. She is the coauthor of two IEEE best paper awards. 


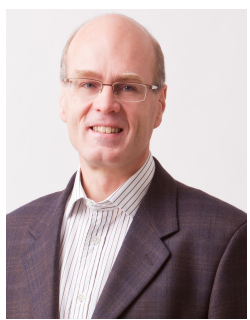

Björn Ottersten (S'87-M'89-SM'99-F'04) was born in Stockholm, Sweden, 1961. He received the M.S. degree in electrical engineering and applied physics from Linkping University, Linkping, Sweden, in 1986. In 1989 he received the Ph.D. degree in electrical engineering from Stanford University, Stanford, CA. Dr. Ottersten has held research positions at the Department of Electrical Engineering, Linköping University, the Information Systems Laboratory, Stanford University, the Katholieke Universiteit Leuven, Leuven, and the University of Luxembourg. During 96/97 Dr. Ottersten was Director of Research at ArrayComm Inc, a start-up in San Jose, California based on Otterstens patented technology. He has co-authored journal papers that received the IEEE Signal Processing Society Best Paper Award in 1993, 2001, 2006, and 2013 and 3 IEEE conference papers receiving Best Paper Awards. In 1991 he was appointed Professor of Signal Processing at the Royal Institute of Technology (KTH), Stockholm. From 1992 to 2004 he was head of the department for Signals, Sensors, and Systems at KTH and from 2004 to 2008 he was dean of the School of Electrical Engineering at KTH. Currently, Dr. Ottersten is Director for the Interdisciplinary Centre for Security, Reliability and Trust at the University of Luxembourg. His research interests include security and trust, reliable wireless communications, and statistical signal processing. 